\title{
Bayesian analysis of multimessenger M-R data with interpolated hybrid EoS
}

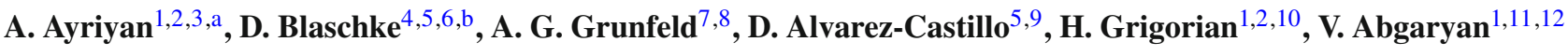 \\ ${ }^{1}$ Laboratory of Information Technologies, JINR, 6 Joliot-Curie Str., Dubna 141980, Russian Federation \\ 2 IT and Computing Division, A. Alikhanyan National Laboratory, 2 Alikhanian Brothers Str., 0036 Yerevan, Armenia \\ ${ }^{3}$ Dubna State University, 19 Universitetskaya Str., Dubna 141980, Russia \\ ${ }^{4}$ Institute of Theoretical Physics, University of Wroclaw, 9 M. Borna Sq, 50-204 Wrocław, Poland \\ ${ }^{5}$ Bogoliubov Laboratory of Theoretical Physics, JINR, 6 Joliot-Curie Str., Dubna 141980, Russian Federation \\ ${ }^{6}$ National Research Nuclear University (MEPhI), 31 Kashirskoe Hwy, Moscow 115409, Russian Federation \\ ${ }^{7}$ CONICET, Godoy Cruz 2290, Buenos Aires, Argentina \\ ${ }^{8}$ Departamento de Física, Comisión Nacional de Energía Atómica, Av. Libertador 8250, (1429), Buenos Aires, Argentina \\ ${ }^{9}$ Henryk Niewodniczański Institute of Nuclear Physics, 152 Radzikowskiego Str, 31-342 Kraków, Poland \\ ${ }^{10}$ Department of Physics, Yerevan State University, 1 Alex Manoogian Str, 0025 Yerevan, Armenia \\ 11 Theoretical Physics Division, A. Alikhanyan National Laboratory, 2 Alikhanian Brothers Str., 0036 Yerevan, Armenia \\ 12 Peoples' Friendship University of Russia (RUDN University), 6 Miklukho-Maklaya Str., Moscow 117198, Russian Federation
}

Received: 26 February 2021 / Accepted: 26 October 2021 / Published online: 29 November 2021

(C) The Author(s) 2021

Communicated by Jerome Margueron

\begin{abstract}
We introduce a family of equations of state (EoS) for hybrid neutron star (NS) matter that is obtained by a two-zone parabolic interpolation between a soft hadronic EoS at low densities and a set of stiff quark matter EoS at high densities within a finite region of chemical potentials $\mu_{H}<\mu<\mu_{Q}$. Fixing the hadronic EoS as the APR one and choosing the color-superconducting, nonlocal NJL model with two free parameters for the quark phase, we perform Bayesian analyses with this two-parameter family of hybrid EoS. Using three different sets of observational constraints that include the mass of PSR J0740+6620, the tidal deformability for GW170817, and the mass-radius relation for PSR J0030+0451 from NICER as obligatory (set 1), while set 2 uses the possible upper limit on the maximum mass from GW170817 as an additional constraint and set 3 instead of the possibility that the lighter object in the asymmetric binary merger GW190814 is a neutron star. We confirm that in any case, the quark matter phase has to be color superconducting with the dimensionless diquark coupling approximately fulfilling the Fierz relation $\eta_{D}=0.75$ and the most probable solutions exhibiting a proportionality between $\eta_{D}$ and $\eta_{V}$, the coupling of the repulsive vector interaction that is required for a sufficiently large maximum mass. We used the Bayesian analysis to investigate with the method of fictitious measure-
\end{abstract}

\footnotetext{
${ }^{a}$ e-mail: alexander.ayriyan@gmail.com

be-mails: david.blaschke@gmail.com; david.blaschke@uwr.edu.pl (corresponding author)
}

ments the consequences of anticipating different radii for the massive $2 M_{\odot}$ PSR J0740+6220 for the most likely equation of state. With the actual outcome of the NICER radius measurement on PSR J0740+6220 we could conclude that for the most likely hybrid star EoS would not support a maximum mass as large as $2.5 M_{\odot}$ so that the event GW190814 was a binary black hole merger.

\section{Introduction}

The observation of the first binary neutron star merger GW170817 in gravitational waves [1] and the subsequent electromagnetic signals from the gamma-ray burst to the light curve of the kilonova [2] have opened the era of multimessenger astronomy. This extends the available mass range for neutron star observations up to $2.6 M_{\odot}$ for the companion star of the $23 M_{\odot}$ black hole in the binary merger GW190814 [3], if that object was indeed the heaviest neutron star and not the lightest black hole, is a currently disputed question. The observation of gravitational waves from the inspiral phase of the merger GW170817 did allow to extract for the first time a new constraint on the equation of state (EoS) of dense matter, the tidal deformability, to be in the range of $70<\Lambda_{1.4}<580$ [4] for a neutron star with the mass of $1.4 M_{\odot}$. From this measurement, together with other constraints, the authors of [5] could constrain the radius of a neutron star in that mass range to the rather narrow limits of $R_{1.4}=11.0_{-0.6}^{+0.9} \mathrm{~km}$. An 
open and controversially discussed question is the interior composition of neutron stars, in dependence of their mass.

It is very likely that the quark substructure of nucleons manifests itself at increasing densities first by a stiffening of the EoS due to quark Pauli blocking in nuclear matter [6] and at still higher densities by a delocalization of the quark wave function and the occurrence of deconfined quark matter. For a recent discussion of soft delocalization vs. hard deconfinement in the transition from nuclear to quark matter, see [7]. A crucial open question, to which the present work intends to contribute, concerns the onset mass of deconfinement and the character of the transition [8].

A standard approach to the hadron-to-quark-matter transition would start from separate EoS models for these two phases and obtain the phase transition from a Maxwell construction (for sufficiently large surface tension between these phases) or a Glendenning construction of a homogeneous mixed phase (for vanishing surface tension) [9]. In-between these limiting cases, the more realistic scenario of the firstorder phase transition would consider structures of finite size formed by the balance between Coulomb interactions and surface tension (pasta phases), see [10] and references therein. This approach has been used recently for a Bayesian analysis with observational constraints for masses and radii of neutron stars [11] which reaches the conclusion that very likely the phase transition onset occurs in the center of neutron stars with masses around $1 M_{\odot}$ and would then match the observed compactness [5] in this way. For this scenario to work, it is customary to have a sufficient stiffness of nuclear matter at supersaturation densities so that the deconfinement transition is driven to relatively low densities.

It is worth noticing that the approach [11] is based on a strong first-order phase transition which entails the formation of hybrid stars as a third family of compact stars [12] featuring the mass twin [13] phenomenon.

In Fig. 1 we illustrate this situation. A soft hadronic EoS like that of Akmal, Pandharipande and Ravenhall (APR) [14] has either no crossing (Maxwell construction) with the color superconducting quark matter EoS (considering a nonlocal version of the NJL model, nINJL, described below) for the weak diquark coupling strength $\left(\eta_{D}=0.71\right)$ or, at slightly increased dimensionless diquark coupling $\left(\eta_{D}=0.79\right)$ an unrealistically early transition that is followed by a "reconfinement" or there is again no transition, depending on the value of the dimensionless vector meson coupling $\eta_{V}$.

In Fig. 2 we show how such pathologies of the phase transition construction (or its inapplicability) with too simple EoS which are not suitable for such a construction, could be cured. A stiffening of the hadronic EoS, here realized by an excluded nucleon volume, leads already to a reasonable transition at not too low densities and to circumvention of the reconfinement problem of a second (unphysical) cross-

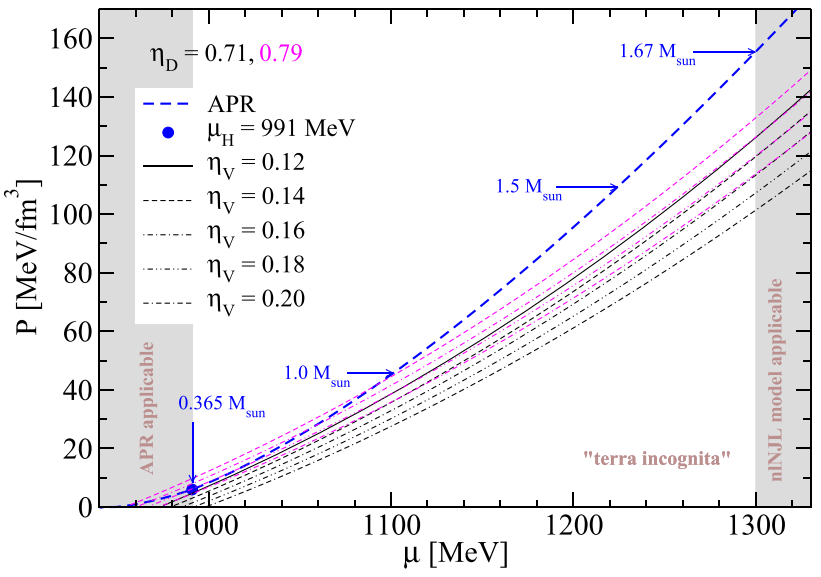

Fig. 1 Pressure vs. chemical potential for the nINJL EoS with different values of $\eta_{V}=0.12(0.02) 0.20$ and the two limiting cases of $\eta_{D}=0.71$ and $\eta_{D}=0.79$ is compared to that of the APR EoS. The point $\mu_{H}$ corresponds here to $n_{H}=1.5 n_{0}$. For orientation, a few selected values of central pressure and chemical potential in neutron stars are indicated by blue arrows labelled with their mass

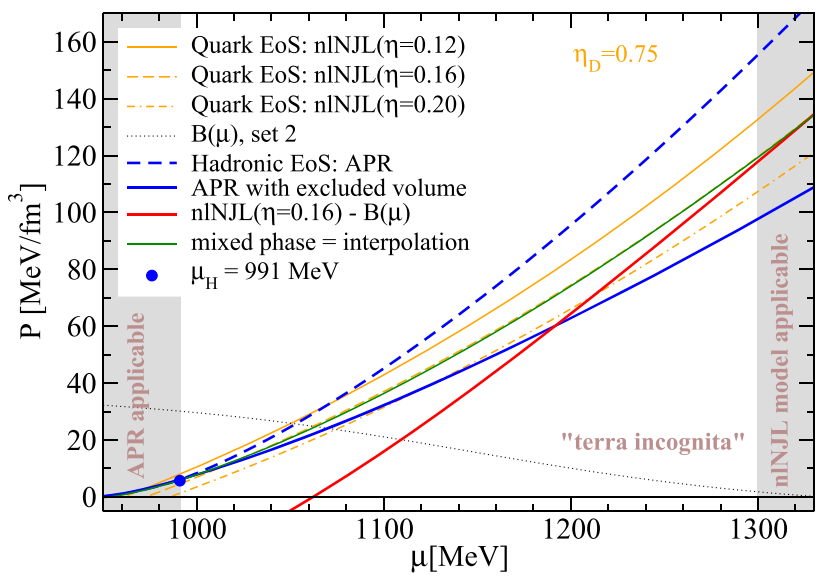

Fig. 2 Pressure vs. chemical potential for the nlNJL EoS (orange lines) with $\eta_{D}=0.75$ and the three values $\eta_{V}=0.12(0.04) 0.20$ compared to that of the APR EoS (dashed blue line) shows that no reasonable Maxwell construction is possible. When a nucleonic excluded volume is applied to APR (solid blue line) and a density-dependent bag pressure $B(\mu)$ according to set 2 of Ref. [15] (black dotted line) to the quark matter EoS, a Maxwell transition point is obtained and a mixed phase construction (green solid line) can be performed which would correspond to an interpolation between APR and nINJL $\left(\eta_{V}=0.16\right)$

ing of hadronic and quark matter EoS at higher densities. ${ }^{1}$ The situation would still be improved towards a more realistic description when confining effects would be included to the quark matter description, e.g., by a (density-dependent) bag pressure that resembles the effect of a nonperturbative QCD vacuum surrounding color charges (quarks) and leads to their confinement in color singlet multiquark states (hadrons). We

${ }^{1}$ For a discussion of the reconfinement problem see, e.g., [16], for the related masquerade problem, see [17] and for their solution see, e.g., [18]. 
note that without such a negative pressure (and/or a confining force), by the larger number of quark and gluon degrees of freedom on the one hand and the larger masses of hadrons in the spectrum on the other, the quark-gluon plasma phase would be favorable over the hadronic matter phase at low temperatures and densities, see Fig. 1 and [19]. As we already noted above, within a first-order phase transition, the formation of structures such a bubbles, droplets, rods and plates (pasta phases) is likely, with their sizes defined by an interplay of surface tension and Coulomb interaction effects.

The resulting pressure (green curve in Fig. 2 looks as if a direct interpolation between the hadronic and the quark matter EoS would have been performed and the underlying three main microphysical ingredients (quark Pauli blocking, quark confinement and pasta structures in the mixed phase) could be circumvented by a direct shortcut from the nuclear matter phase just above saturation density to the quark matter phase which would then appear as a crossover-like EoS.

Such crossovers have been invoked on physical grounds by symmetry arguments as a quark-hadron continuity in Refs. [20-22] and by the combined effects of chiral symmetry breaking and diquark condensation intertwined by the axial anomaly so that they result in a crossover at low temperatures which eventually entails a second critical endpoint in the QCD phase diagram [23-25]. The crossover behaviour has subsequently been realized in effective interpolating constructions following [26-29] and further literature in this direction.

While in interpolating constructions the information about the composition of the matter in the mixed phase is lost, they allow for conclusions on the likely properties of the pure quark matter EoS that is used as input, once this phase is reached in neutron star interiors. In contrast, the dominant class of EoS used to extract EoS constraints from observations of masses and radii [30-32] as well as tidal deformabilities [33,34] and in near future also the moment of inertia [35] are the multi-polytrope extrapolations of the EoS at supersaturation densities. Here we referred only to some prominent examples. These constraints have also been used to perform Bayesian analyses of the most likely EoS, see for instance the recent work of Miller et al. [36]. We note that analyses based on the multi-polytrope ansatz for the high-density EoS are agnostic of the composition of matter as well as constraints on the microphysics of dense quark matter.

In this paper, we will perform a Bayesian analysis study with modern mass and radius constraints, as well as fictitious radius measurements, on the basis of a new, two-zone interpolation construction for obtaining hybrid EoS that allows for conclusions on the most favorable parameter set for the lagrangian model of color superconducting quark matter with nonlocal chiral interactions of the Nambu-Jona-Lasinio type. The EoS model, including the new interpolation procedure is described in the next section. The results for masses, radii and tidal deformabilities of hybrid stars are outlined in Sect. 3 and astrophysical inputs for the Bayesian analyses are given in Sect. 4. The results of the Bayesian analysis are presented in Sect. 6 and in Sect. 7 we draw the conclusions from this study.

\section{New class of quark-hadron hybrid EoS by two-zone interpolation}

The idea is to interpolate between hadron and quark EoS models from the trustable region of the hadronic EoS at the nuclear saturation density $\left(n_{H}=1.0 \ldots 1.5 n_{0}\right)$ to the trustable region for the quark matter model at $n_{Q} \gtrsim 3 n_{0}$ (see Fig. 1). Before we outline in detail the new interpolation method in Sect. 2.3, we specify in the following two subsections the hadronic and the quark matter EoS that we employ in the present study to describe the pure phases outside the region of "terry incognita" indicated in Figs. 1 and 2.

\subsection{Hadronic EoS}

Our choice of hadronic equation of state for this work is the well known APR model [14]. It is a non-relativistic model derived by means of variational chain summation methods which included Urbana potentials of two and three nucleon interactions and features a pion condensate. Moreover, it exhibits a causality breach in neutron star matter for massive stars, a problem that shall not appear for the hybrid star models build in this work. The APR EoS version we have chosen is $\mathrm{A} 18+\delta \mathrm{v}+\mathrm{UIX}^{*}$ which is not extremely stiff, reaching the maximum neutron star mass right below $2 \mathrm{M}_{\odot}$.

In addition, in order to complete the description of the neutron star matter EoS, we adjoin a low density region EoS corresponding to the crust of neutron stars, namely the SLy4 model [37].

\subsection{Quark matter EoS}

For the description of the quark matter phase we consider a nonlocal chiral quark model, as in Ref. [15], which includes scalar quark-antiquark interaction, anti-triplet scalar diquark interactions and vector quark-antiquark interactions. The grand canonical thermodynamic potential per unit volume at zero temperature and finite density in the mean field approximation (MFA) reads

$$
\begin{aligned}
\Omega^{\mathrm{MFA}}= & \frac{\bar{\sigma}^{2}}{2 G_{S}}+\frac{\bar{\Delta}^{2}}{2 H}-\frac{\bar{\omega}^{2}}{2 G_{V}} \\
& -\frac{1}{2} \int \frac{d^{4} p}{(2 \pi)^{4}} \ln \operatorname{det}\left[S^{-1}\left(\bar{\sigma}, \bar{\Delta}, \bar{\omega}, \mu_{f c}\right)\right],
\end{aligned}
$$


see Ref. [38] for details of the calculation. The input parameters of the model are determined as to reproduce meson properties in the vacuum, at vanishing temperature and densities, then, $m_{c}, p_{0}$ (the cutoff) and $G_{S}$ can be determined under that conditions. The remaining coupling constants $G_{D}$ and $G_{V}$ are driving the terms that, after bosonization, give rise to the superconducting gap field and the vector field. Then, the ratios $\eta_{D}=G_{D} / G_{S}$ and $\eta_{V}=G_{V} / G_{S}$ are input parameters. For OGE interactions in the vacuum, Fierz transformation leads to $\eta_{D}=3 / 4$ and $\eta_{V}=1 / 2$. As the microscopic interaction is not derived directly from QCD then, the above coupling ratios have in principle no strong phenomenological constraint except for the fact that $\eta_{D}$ values larger than $\eta_{D}^{*}=(3 / 2) m /\left(m-m_{c}\right)$ may lead to color symmetry breaking in the vacuum [39] (where $m$ stands for the dressed mass and $m_{c}$ for the current quark mass). In the present work we consider $\eta_{D}$ and $\eta_{V}$ as free parameters to be varied in reasonable limits, as it has been done for the NJL model case in Ref. [40]. The mean field values $\bar{\sigma}, \bar{\Delta}$ and $\bar{\omega}$ satisfy the coupled equations

$\frac{d \Omega^{\mathrm{MFA}}}{d \bar{\Delta}}=0, \quad \frac{d \Omega^{\mathrm{MFA}}}{d \bar{\sigma}}=0, \quad \frac{d \Omega^{\mathrm{MFA}}}{d \bar{\omega}}=0$.

As we are focused on describing the behaviour of quark matter in the core of NSs, we have to impose: equilibrium under weak interactions, chemical equilibrium, and color and electric charge neutrality. Then, the six different chemical potentials $\mu_{f c}$ in Eq. (1) (depending on the two quark flavors $u$ and $d$ and quark colors $r, g$ and $b$ ), can be written in terms of three independent quantities: the baryonic chemical potential $\mu$, the electron chemical potential $\mu_{e}$ and a color chemical potential $\mu_{8}$. So basically, for each value of $\mu$ we selfconsistently solve the gap equations (2), complemented with the conditions for $\beta$-equilibrium and electric charge and color charge neutrality (details of the calculation can be found in the Appendix of Ref. [38]).

In the present work, we consider a Gaussian form factor $g(p)=\exp \left(-p^{2} / p_{0}^{2}\right)$ in Euclidean 4-momentum space. The input parameters of the quark model are fixed to $m_{c}=5.4869$ $\mathrm{MeV}, p_{0}=782.16 \mathrm{MeV}$ and $G_{S} p_{0}^{2}=19.804$ so that the pion mass $m_{\pi}=139 \mathrm{MeV}$, the pion decay constant $f_{\pi}=92.4$ $\mathrm{MeV}$ and the chiral condensate $-\langle\bar{q} q\rangle^{1 / 3}=244 \mathrm{MeV}$ are reproduced. As mentioned above, we perform our analysis considering $\eta_{D}$ and $\eta_{V}$ as free parameters.

\subsection{Two-zone interpolation method}

We introduce here a two-zone interpolation scheme that is inspired by the discussion in subsection V. D (Fig. 15) and V. G (Fig. 18) of Ref. [8], see also Fig. 2 and its discussion in the previous section. According to that discussion, the interpolated part of the hybrid EoS can be motivated as a result of hadronic interactions and many-body forces
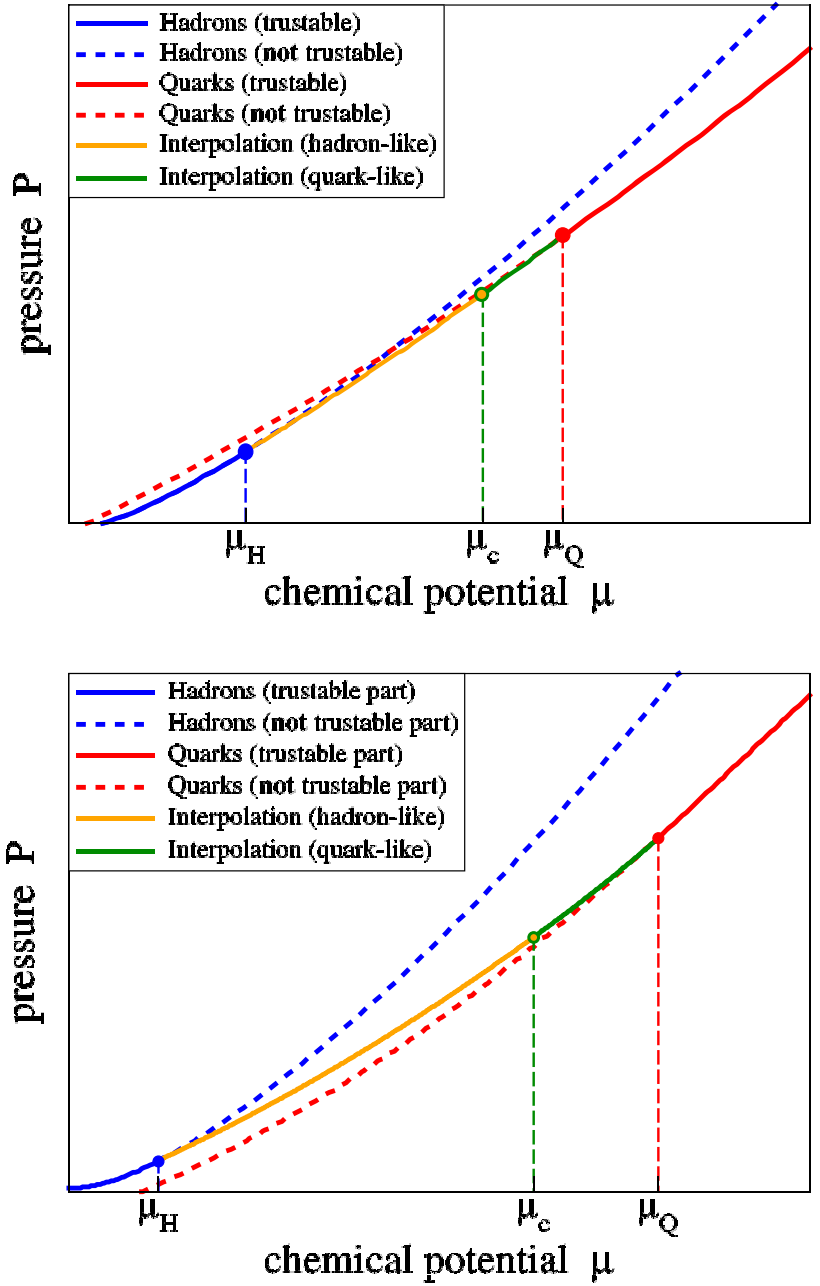

Fig. 3 A hybrid equation of state that joins a soft nuclear equation of state with a stiffer quark matter equation of state by interpolation in the intermediate region between $\mu_{H}$ and $\mu_{Q}$ for $n\left(\mu_{H}\right)=n_{H} \sim n_{0}$ and $n\left(\mu_{Q}\right)=n_{Q} \sim(2.5 \ldots 5) n_{0}$. The dotted curves indicate where the extrapolations of the nuclear and quark matter equations of state become unreliable. We can treat the case where these extrapolations cross each other (upper panel) as well as the case when they do not cross (lower panel)

in the hadronic phase (leading to $P_{H}(\mu) \rightarrow P_{H}^{*}(\mu)$ ) and confining effects in the quark matter phase (resulting in $\left.P_{Q}(\mu) \rightarrow P_{Q}^{*}(\mu)\right)$. In general, one would have to expect that these effects lead to a "normal" crossing of hadronic and quark matter $P(\mu)$ curves, but with a "kink" at the location $\mu_{c}$ of the crossing point which results in a jump the density $\Delta n\left(\mu_{c}\right)=d P_{Q}^{*} /\left.d \mu\right|_{\mu_{c}}-d P_{H}^{*} /\left.d \mu\right|_{\mu_{c}}>0$. In this work we discuss the continuous interpolation as the limiting case for $\Delta n\left(\mu_{c}\right) \rightarrow 0$.

An advantage of the two-zone interpolation between $n_{H}$ and $n_{Q}$ over a simple single-zone interpolation is that one can obtain good results without employing higher-order polyno- 
mials [41,42], just by using two parabolic functions

$$
\left\{\begin{array}{l}
P_{\eta}(\mu)=a_{\eta}\left(\mu-\mu_{H}\right)^{2}+b_{\eta}\left(\mu-\mu_{H}\right)+c_{\eta}, \mu \leq \mu_{c}, \\
P_{\rho}(\mu)=a_{\rho}\left(\mu-\mu_{Q}\right)^{2}+b_{\rho}\left(\mu-\mu_{Q}\right)+c_{\rho}, \mu \geq \mu_{c}
\end{array}\right.
$$

where $\mu_{H}$ and $\mu_{Q}$ correspond to $n_{H}$ and $n_{Q}$ respectively, and $\mu_{c}$ is free parameter taking value between them: $\mu_{H}<$ $\mu_{c}<\mu_{Q}$.

The four parameters $b_{\eta}, b_{\rho}, c_{\eta}$ and $c_{\rho}$ can be immediately defined from the boundary conditions

$$
\begin{aligned}
P_{\eta}\left(\mu_{H}\right) & \equiv P_{H}\left(\mu_{H}\right)=c_{\eta}, \\
n_{\eta}\left(\mu_{H}\right) & \equiv n_{H}\left(\mu_{H}\right)=b_{\eta}, \\
P_{\rho}\left(\mu_{Q}\right) & \equiv P_{Q}\left(\mu_{Q}\right)=c_{\rho}, \\
n_{\rho}\left(\mu_{Q}\right) & \equiv n_{Q}\left(\mu_{Q}\right)=b_{\rho} .
\end{aligned}
$$

The remaining parameters $a_{\eta}, a_{\rho}$ are defined by the matching conditions at at the intermediate $\mu_{c}$ where both functions should be sewed

$$
\left\{\begin{array}{l}
P_{\eta}\left(\mu_{c}\right)=P_{\rho}\left(\mu_{c}\right) \\
n_{\eta}\left(\mu_{c}\right)=n_{\rho}\left(\mu_{c}\right)-\Delta n\left(\mu_{c}\right)
\end{array}\right.
$$

These conditions are equivalent to the following system of linear algebraic equations (SLAE)

$$
\left\{\begin{array}{l}
a_{\eta}\left(\mu_{c}-\mu_{H}\right)^{2}-a_{\rho}\left(\mu_{c}-\mu_{Q}\right)^{2}=\kappa_{1}, \\
2 a_{\eta}\left(\mu_{c}-\mu_{H}\right)-2 a_{\rho}\left(\mu_{c}-\mu_{Q}\right)=\kappa_{2},
\end{array}\right.
$$

where

$$
\left\{\begin{array}{l}
\kappa_{1}=n_{Q}\left(\mu_{c}-\mu_{Q}\right)-n_{H}\left(\mu_{c}-\mu_{H}\right)+P_{Q}-P_{H}, \\
\kappa_{2}=n_{Q}-n_{H}-\Delta n\left(\mu_{c}\right) .
\end{array}\right.
$$

The determinant of this SLAE

$$
\Delta=2\left(\mu_{c}-\mu_{Q}\right)\left(\mu_{c}-\mu_{H}\right)\left(\mu_{H}-\mu_{Q}\right)
$$

shows that the system has always a solution when $\mu_{c} \neq \mu_{Q}$, $\mu_{c} \neq \mu_{H}$ and $\mu_{H} \neq \mu_{Q}$. The solution to the SLAE is

$$
\left\{\begin{array}{l}
a_{\eta}=\frac{-2 \kappa_{1}+\kappa_{2}\left(\mu_{c}-\mu_{Q}\right)}{2\left(\mu_{c}-\mu_{H}\right)\left(\mu_{H}-\mu_{Q}\right)}, \\
a_{\rho}=\frac{-2 \kappa_{1}+\kappa_{2}\left(\mu_{c}-\mu_{H}\right)}{2\left(\mu_{c}-\mu_{Q}\right)\left(\mu_{H}-\mu_{Q}\right)} .
\end{array}\right.
$$

We note, that this two-zone interpolation allows for a generalization to describe a first-order phase transition. This could be achieved by choosing a nonzero value for the jump in the density $\Delta n\left(\mu_{c}\right)$, which appears as additional parameter in the second equation of the system (8).

\subsection{Constant speed-of-sound representation}

For the nINJL model EoS following from (1), a causality violation at high energy densities (which corresponds to very high chemical potentials) appears due to a backbending of the quark pressure as a function of the energy density. To circumvent that obstacle, we make use of the recently discovered fact that in the range of densities relevant for NS applications the nlNJL model can be represented with high accuracy by a constant-speed-of-sound (CSS) EoS [43]. This EoS is given by $[44,45]$

$$
\left\{\begin{array}{l}
P(\mu)=P_{0}+P_{1}\left(\frac{\mu}{\mu_{x}}\right)^{1+1 / c_{s}^{2}}, \\
\varepsilon(\mu)=-P_{0}+P_{1} \frac{1}{c_{s}^{2}}\left(\frac{\mu}{\mu_{x}}\right)^{1+1 / c_{s}^{2}}, \\
n_{B}(\mu)=P_{1} \frac{1+1 / c_{s}^{2}}{\mu_{x}}\left(\frac{\mu}{\mu_{x}}\right)^{1 / c_{s}^{2}} .
\end{array}\right.
$$

Here $\mu_{x}$ is a scale for the chemical potential which we set to $\mu_{x}=1 \mathrm{GeV}$ in correspondence with $[43,45]$. The parameters $P_{0}, P_{1}$ and $c_{s}^{2}$ are obtained from the parameters $\eta_{D}$ and $\eta_{V}$ of the nINJL model by a functional mapping that has been defined in Ref. [43]. We note that the values for the squared sound speed obtained by this fit are in the range $c_{s}^{2}=0.45 \ldots 0.54$.

\subsection{Hybrid EoS from a two-zone interpolation}

The interpolation method has been implemented for APR and NJL models with different $\eta_{D}=0.71(0.02) 0.79$ and $\eta_{V}=0.06(0.02) 0.20$ (see Figs. 4, 5, 6, 7 and 8). The onset density for the interpolation has been set to $n_{H}=n_{0}$, and the density where the interpolation matches the quark matter EoS has been varied depending on $\eta_{V}$ as $n_{Q}=4.5 \ldots 2.5 n_{0}$ while simultaneously $\eta_{V}$ was incremented. The value of $\mu_{c}$ has been fixed as $\mu_{c}=\mu_{H}+0.75\left(\mu_{Q}-\mu_{H}\right)$.

It is interesting to observe in Figs. 7 and 8 the similarity in the behaviour of the squared speed of sound with that of recent models of quarkyonic compact star matter [46]. A fast rise (stiffening of nuclear matter) is followed by a dip (hadron-quark mixed phase) and then saturates at an asymptotic value. This value in our case is the value obtained for the CSS fit of the nINJL model and ranges between 0.45 and 0.54 . Since the present neutron star phenomenology requires a stiffness of the EoS that results in a maximum mass of at least $2 M_{\odot}$, the corresponding energy densities that are probed by neutron star interiors do not exceed about $1 \mathrm{GeV} / \mathrm{fm}^{3}$, see Fig. 9. This means that with the present setting of the twozone interpolation construction, the pure quark matter phase for $n_{B}>n_{Q}$ is barely reached in stable neutron star configurations, if at all. 


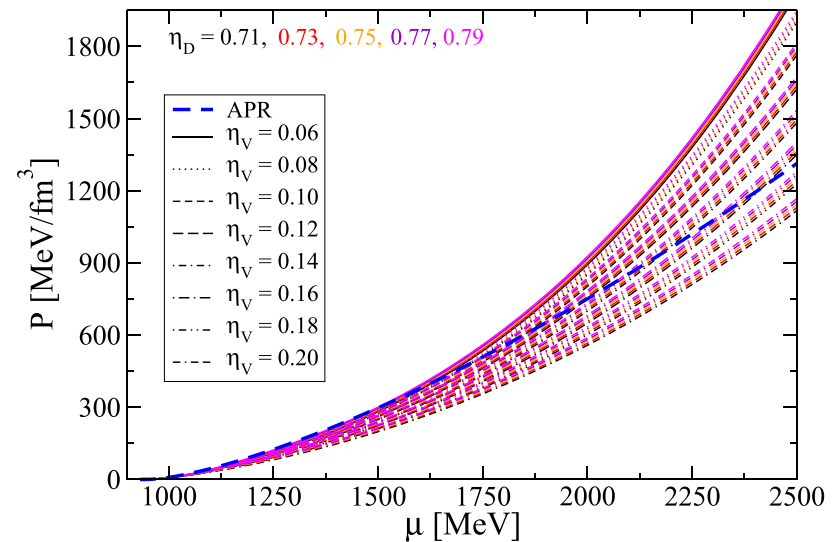

Fig. 4 Two-zone interpolation construction between APR and nINJL on $P-\mu$ plot

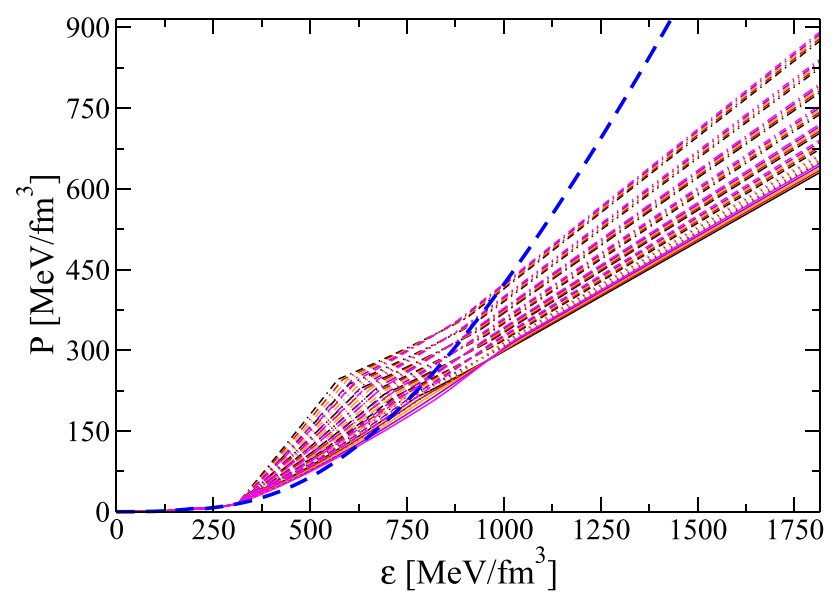

Fig. 5 Two-zone interpolation construction between APR and nINJL on $P-\varepsilon$ plot. Legend as in Fig. 4

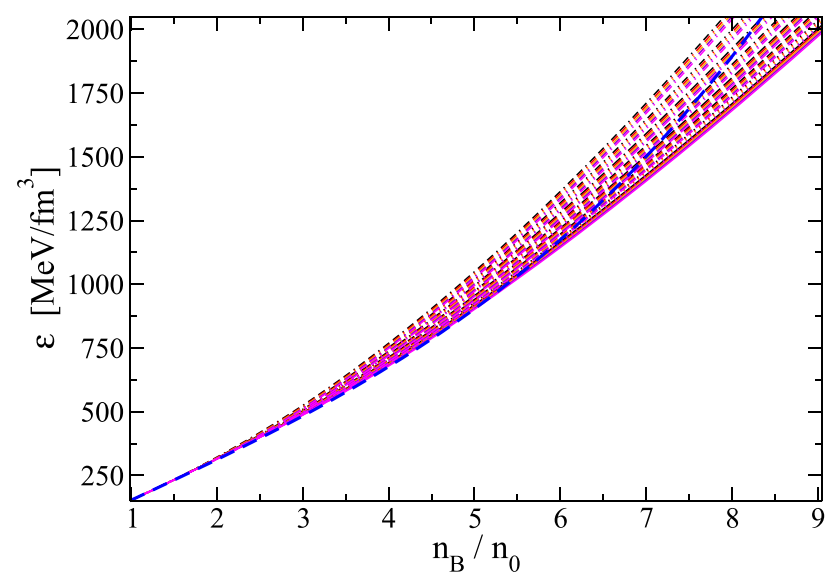

Fig. 6 Energy density dependence on the baryon density for the EoS curves under consideration. Legend as in Fig. 4

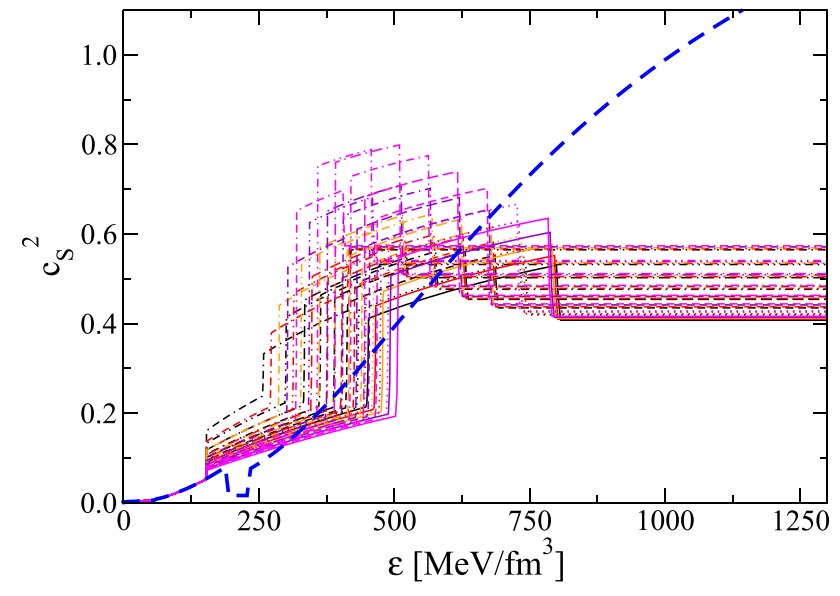

Fig. 7 Speed of sound vs energy density for the two-zone interpolation construction between APR and nlNJL. Legend as in Fig. 4

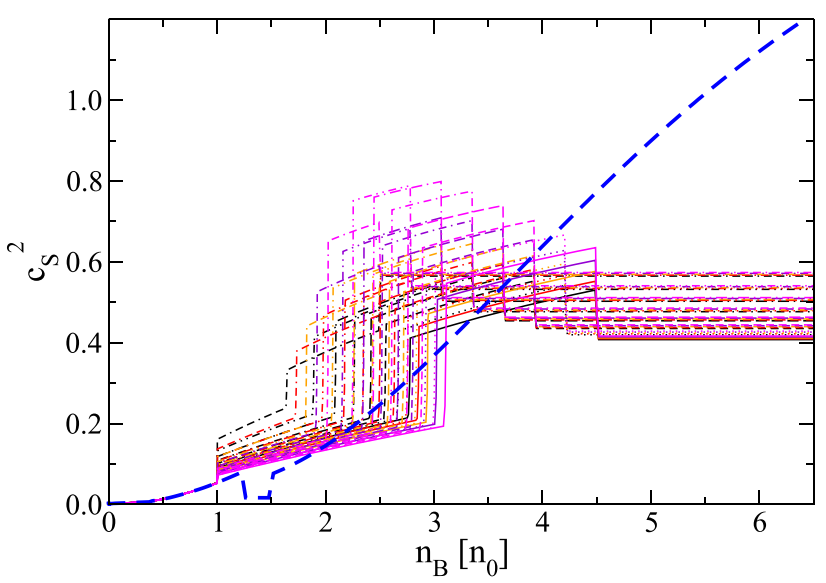

Fig. 8 Speed of sound vs. density for the two-zone interpolation construction between APR and the set of nlNJL quark matter EoS from Fig. 5. Legend as in Fig. 4

\section{Masses, radii and tidal deformabilities for the hybrid EoS}

Neutron stars are computed within the framework of general relativity by using the corresponding EoS in the form of $p(\varepsilon)$ to solve the Tolman-Oppenheimer-Volkoff (TOV) equations $[47,48]$

$$
\begin{aligned}
\frac{\mathrm{d} p(r)}{\mathrm{d} r} & =-\frac{(\varepsilon(r)+p(r))\left(m(r)+4 \pi r^{3} p(r)\right)}{r(r-2 m(r))}, \\
\frac{\mathrm{d} m(r)}{\mathrm{d} r} & =4 \pi r^{2} \varepsilon(r) .
\end{aligned}
$$

which describe a static, spherical star. Radial mass $m(r)$, energy density $\varepsilon(r)$ and pressure $p(r)$ stellar internal profiles help to determined the mass $M$ and radius $R$ of a star with central density $\varepsilon_{c}$ with boundary conditions $m(r=0)=0$ and $p(r=R)=0$. In Figs. 9 and 10 we show the compact star mass as a function of the central energy density 


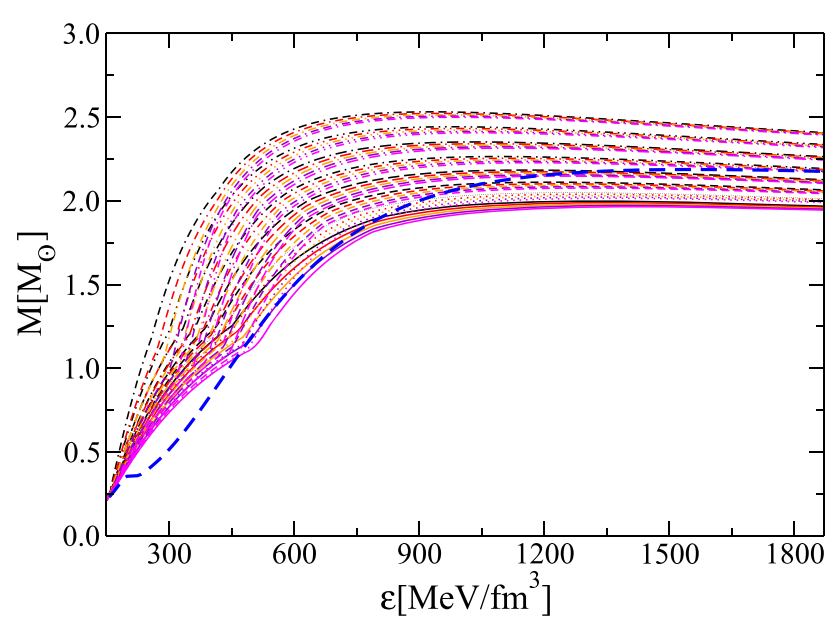

Fig. 9 Mass vs. central energy density for the two-zone interpolation construction between APR and the set of nlNJL quark matter EoS. Legend as in Fig. 4

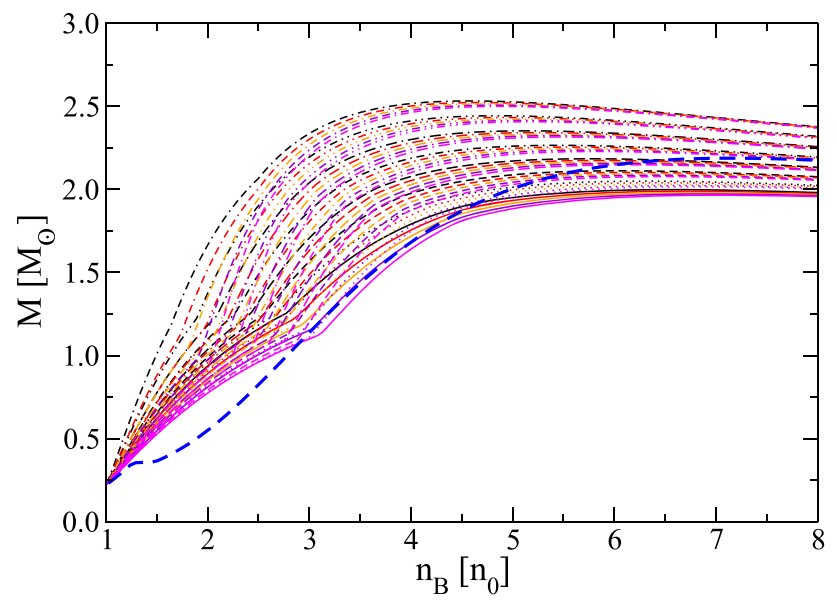

Fig. 10 Mass vs. central baryon density for the two-zone interpolation construction between APR and the set of nINJL quark matter EoS. Legend as in Fig. 4

and baryon density, respectively, for the two-zone interpolation construction. The dashed blue line corresponds to the hadronic APR EoS and the remaining curves show the hybrid EoS for a range of input parameters for the quark matter phase (same patters/colors as in previous figures). The compact star mass-radius sequence is a benchmark for every EoS model commonly presented in a mass-radius diagram that includes pulsar measurement regions as well as excluded ones by other astrophysical observations, see for instance Fig. 11. The EoS sequences displayed in there are obtained by a systematic integration of the the TOV equations for increasing $p_{c}$ for each single star up to the value of the maximum mass for which the condition $\partial M / \partial \varepsilon_{c}>0$ holds.

In addition, deformation of the compact star is a feature of the EoS that is closely related to the last moments of the inspiral phase of compact star mergers. It is quantified by

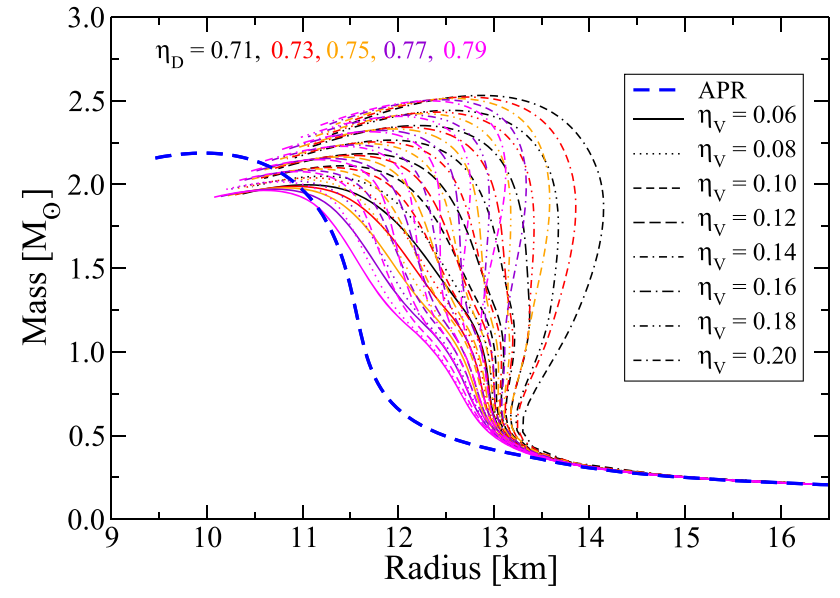

Fig. 11 Mass-radius relations for the two-zone interpolation construction between APR and nlNJL

computing the tidal deformability $\Lambda$, for which estimated regions were derived by the observation of the GW170817 event $[1,4]$, also displayed in Fig. 11. The corresponding equations are derived from perturbations of the spherical metric of the compact star supplemented with the stellar internal profiles for physical quantities derived from the TOV equations. The equation

$\Lambda=\frac{2}{3} \frac{R^{5}}{M^{5}} k_{2}$

relates the dimensionless tidal deformability $\Lambda$ with the tidal Love number $k_{2}$ and the total mass and radius of the star. Details on the derivation of the above formula can be found in [49-53].

\section{Bayesian inference}

In this section we introduce the Bayesian methodology and its application to the set of considered EoS characterized by the parameters $\eta_{D}$ and $\eta_{V}$ in order to find their best values that fulfill observational data.

The aposteriori probability $P\left(\pi_{q} \mid E\right)$ is a conditional probability of the given vector of parameters $\pi_{q}$ (introduced below), where $q$ denotes the indexes the values of parameters for each alliterative representation of the model of EoS. The condition $E$ is the set of the observational data (events), its likelihood for the given model is represented as a product

$P\left(E \mid \pi_{q}\right)=\prod_{\alpha} P\left(E_{\alpha} \mid \pi_{q}\right)$,

which is the the conjunction of all events $E_{\alpha}$ (where $\alpha$ is an index of an event). The a posteriori probabilities, likelihoods and a priori probabilities are connected to each other via the 
Bayes formula

$$
P\left(\pi_{q} \mid E\right)=\frac{P\left(E \mid \pi_{q}\right) P\left(\pi_{q}\right)}{\sum_{p=0}^{N-1} P\left(E \mid \pi_{p}\right) P\left(\pi_{p}\right)},
$$

where the factor $P\left(\pi_{q}\right)$ is the prior of a given model.

First, we define the set of values of the parameters $\eta_{D}$ and $\eta_{V}$ as

$H_{D}=\{0.71,0.73,0.75,0.77,0.79\}$

and

$H_{V}=\{0.06,0.08,0.10,0.12,0.14,0.16,0.18,0.20\}$.

Then, the vector of parameters $\pi_{q}$ will be an element of the set $H_{D} \times H_{V}$,

$\pi_{q} \in\left\{\eta_{D(i)}, \eta_{V(j)} \mid i=0 . . N_{D}-1, j=0 . . N_{V}-1\right\}$,

where $q=i N_{V}+j$ and $N_{V}=8, N_{D}=5$. Therefore, $q=0 . . N-1$ and $N=40$ is the full number of model representations. For the choice of the uniform distribution of the prior we have

$P\left(\pi_{q}\right)=1 / N$.

In the next section, we discuss the specific astrophysical constraints that we will employ in our Bayesian analysis (BA).

\section{Astrophysical constraints}

\subsection{Lower limit of maximum mass}

Recently, the mass of the PSRJ0740+6620 was obtained recently by a Shapiro delay based measurement combining data from the North American Nanohertz Observatory for Gravitational Waves (NANOGrav) and data from the orbital-phase-specific observations using the Green Bank Telescope. The $68.3 \%$ of the credibility interval was given as $2.14_{-0.09}^{+0.10} M_{\odot}$ in [54], but it has recently been updated to $2.08 \pm 0.07 M_{\odot}$ in [55]. This value has been chosen as the lower limit of maximum mass $\left(M_{\mathrm{max}}^{\mathrm{low}}\right)$.

The likelihood for the lower limit of maximum mass constraint is given by

$P\left(E_{M} \mid \pi_{q}\right)=\Phi\left(M_{q}, \mu_{l}, \sigma_{l}\right)$,

where $M_{q}$ is the maximum mass of the sequence of neutron star configurations for the given $\pi_{q}$, and $\Phi(M, \mu, \sigma)$ is the cumulative distribution function (CDF) of the standard normal distribution. And $\mu_{l}$ and $\sigma_{l}$ are the parameters of the uncertainty of a low limit maximum mass estimation.

Additionally, the assumption that one of the component of the binary merger GW190814 is a neutron star gives an estimation of the lower limit of the maximum mass as
$2.59_{-0.09}^{+0.08} M_{\odot}$ [3]. This value has been used in the Bayesian Inference as an alternative scenario for the lower limit. In Fig. 12 we display the mass-radius relations for our hybrid configurations together with the APR model as the low density baseline for hadronic matter that becomes invalid at higher densities where it crosses over to the nINJL quark matter model. The different colored regions correspond to either mass and/or radius measurements or to forbidden regions following from GW170817 phenomenology that serve as constraints for the compact star EoS. The horizontal black dashed lines show the mass range for the lighter object in the binary merger GW190814, that we employ as a possible lower limit on the maximum mass, in the case that this object was a neutron star.

\subsection{Upper limit of maximum mass}

There is an estimation of the upper limit of maximum mass of neutron star in the literature [60]. It was estimated with combination of the observation of gravitational waves (GW170817) and drawing from basic arguments on kilonova modeling of GRB 170817A, together with the quasiuniversal relation between the maximum masses of static neutron stars and the fastest stable star under uniform rotation [62]. The upper limit of the maximum mass is $2.16_{-0.15}^{+0.17} M_{\odot}$ as shown in Fig. 12.

The likelihood for the upper limit of maximum mass constraint is given by

$P\left(E_{M} \mid \pi_{q}\right)=1-\Phi\left(M_{q}, \mu_{u}, \sigma_{u}\right)$

where $M_{q}$ is the maximum mass of the sequence of neutron star configurations for the given $\pi_{q}$, and $\mu_{u}=2.16 M_{\odot}$ and $\sigma_{u}=0.17 M_{\odot}$.

However, in Ref. [61] a relationship between the onset mass of prompt collapse to a black hole, the tidal deformability at half this mass and the maximum TOV mass has been derived, according to which the fact that the merger GW170817 did not promptly collapse to a black hole implies a lower limit on the maximum TOV mass. Therefore, we will include the disputable maximum mass constraint of Ref. [60] only in one of the sets of our Bayesian analysis.

\subsection{Gravitational wave constraint}

The observation of the gravitational waves from binary NSNS merger GW170817 allows to calculate relation between tidal deformabilities of the primary and secondary components $[1,4]$. In order to implement the tidal deformability constraint to Bayesian Inference the Gaussian kernel density estimation has been used to recover the probability distribu- 


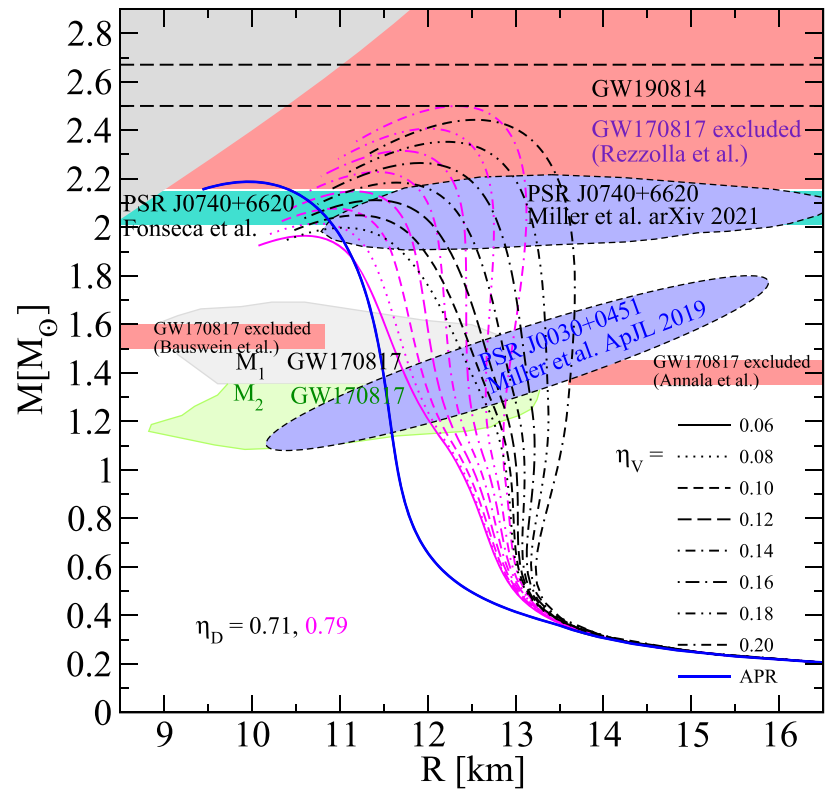

Fig. 12 Mass-radius relations for EoS models featuring an interpolation scheme [8] between the low density APR model for hadronic matter and high density nlNJL quark matter. A few compact star sequences are displayed for two fixed quark matter parameter values of $\eta_{D}$ with varying $\eta_{V}$ together with the hadronic APR EoS. Different color regions correspond to either pulsar measurements or forbidden regions that serve as constraints for the compact star EoS. The green band region above $2 \mathrm{M}_{\odot}$ corresponds to the updated mass measurement of PSR J0740+6620 [55], which was recently upgraded to a mass-radius measurement by NICER, shown by the blue ellipsoidal region for the result of the MarylandIllinois team [56]. The other blue ellipse corresponds to the mass and radius measurement of PSR J0030+0451 by NICER [58] whereas the grey and light green regions correspond to the estimates of the components of the binary system labeled as $M_{1}$ and $M_{2}$ of the GW170817 merger [4]. Red bands correspond to excluded regions derived from GW170817 observations by Bauswein et al. [59], Annala et al. [33] and Rezzolla et al. [60]. The black dashed horizontal lines are the upper and lower limit for the mass $2.59_{-0.09}^{+0.08} M_{\odot}$ of the lighter component in the binary merger event GW190814 [3]

tion function with use of the data on the $\Lambda_{1}-\Lambda_{2}$ publicly shared by LIGO collaboration. ${ }^{2}$

The likelihood of the gravitational wave constraint is introduced as

$P\left(E_{G W} \mid \pi_{q}\right)=\int_{l} \beta\left(\Lambda_{1}\left(n_{c}\right), \Lambda_{2}\left(n_{c}\right)\right) \mathrm{d} n_{c}$,

where $l$ is the length of the line on the $\Lambda_{1}-\Lambda_{2}$ diagram produced by $\vec{\pi}_{q}$, and $n_{c}$ is the central baryon density of a star. $\beta\left(\Lambda_{1}, \Lambda_{2}\right)$ is the probability distribution function (PDF) that has been reconstructed (as previously done in $[63,64]$ ). In Fig. 13 we show the dimensionless tidal deformabilities of hybrid compact stars configurations. The line colors/patters are the same as in previous figures. We display as well the corresponding measurement from GW170817. Figure 14 shows

\footnotetext{
${ }^{2}$ https://dcc.ligo.org/LIGO-P1800115/public.
}

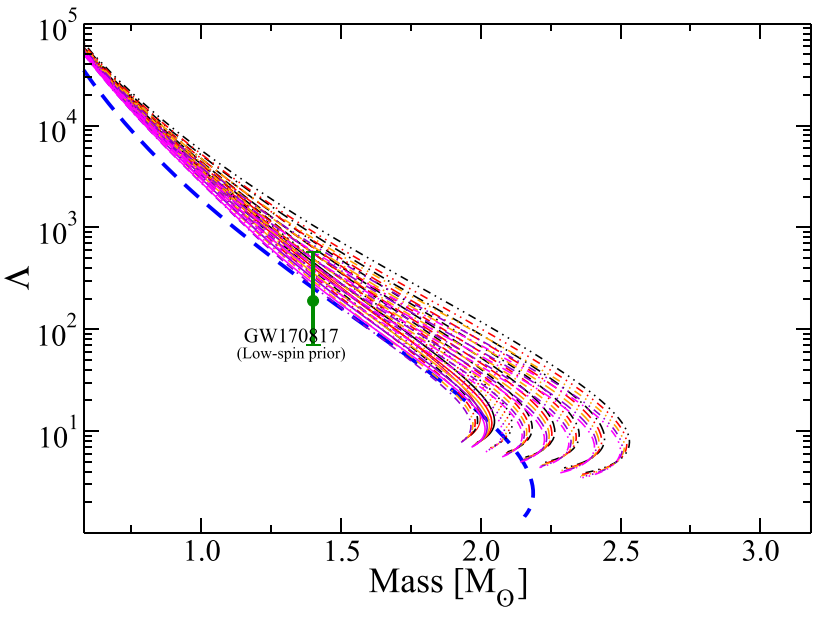

Fig. 13 Dimensionless tidal deformabilities of hybrid compact stars together with the corresponding measurement from GW170817 [4]

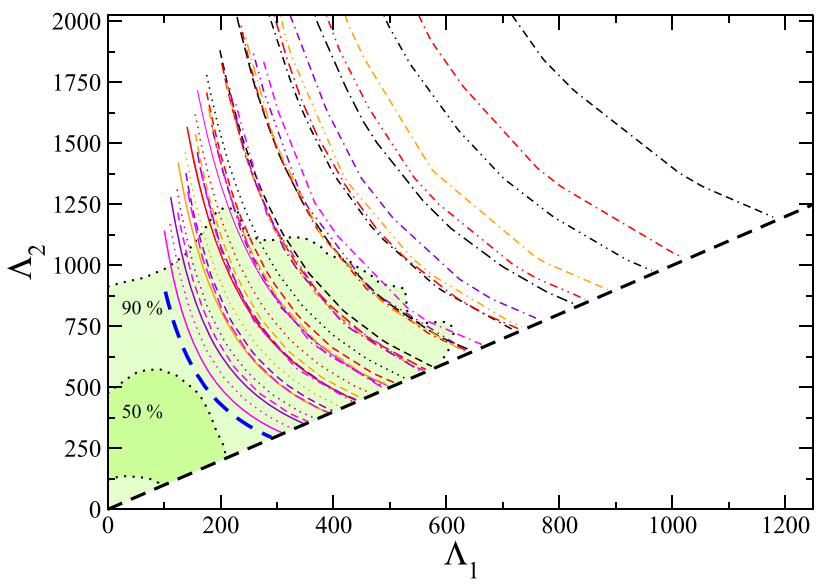

Fig. 14 Tidal deformabilities diagram. $\Lambda_{1}$ and $\Lambda_{2}$ correspond to the dimensionless tidal deformability for each of the components of the binary in GW170817. Light and dark green regions correspond to the $50 \%$ and $90 \%$ credibility regions for the posteriors used in the LIGOVirgo analysis [4]. All the EoS in this work result in curves that fall inside the lighter region as well as the APR EoS which is displayed in blue

our results for the tidal deformabilities $\Lambda_{2}$ as a function of $\Lambda_{1}$. Dark and light green regions correspond to the $50 \%$ and $90 \%$ credibility for the posteriors used in the LIGO-Virgo Collaboration analysis, respectively. All the hybrid EoS in this work result in curves that fall inside the $90 \%$ region as the APR EoS which is displayed as dashed blue line.

\subsection{Mass-radius constraint}

A simultaneous measurement of mass and radius has been performed use data collected by the Neutron Star Interior Composition Explorer (NICER) space observatory for the 
pulsar PSR J0030+0451. The results of the observation have been reported in a collection of publications. ${ }^{3}$

There were two independent analysis of the mass and equatorial radius based on mutually exclusive assumptions about the uniform-temperature emitting spots. The first result for radius and mass is $M_{1}=1.44_{-0.14}^{+0.15} M_{\odot}$ and $R_{1}=13.02_{-1.06}^{+1.24} \mathrm{~km}$ [58] whereas the second one is $M_{2}=$ $1.34_{-0.16}^{+0.15} M_{\odot}$ and $R_{2}=12.71_{-1.19}^{+1.14} \mathrm{~km}$ [65]. A bivariate probability distribution function $\alpha(M, R)$ has been reconstructed by the method of the Gaussian kernel density estimation using the data [66]. A likelihood is formulated as

$P\left(E_{G W} \mid \pi_{q}\right)=\int_{l} \alpha\left(M\left(n_{c}\right), R\left(n_{c}\right)\right) \mathrm{d} n_{c}$,

where $M\left(n_{c}\right)$ and $R\left(n_{c}\right)$ are mass and radius of sequence of neutron star for a given $q$ th equation of state, and $n_{c}$ is the central baryon density.

Another simultaneous measurement of the mass and the equatorial radius has recently been published based on NICER observations combined with XMM Newton data of PSR J0740+6620. The two independent analysis teams reported $R=13.7_{-1.5}^{+2.6} \mathrm{~km}[56]$ and $R=12.39_{-0.98}^{+1.3} \mathrm{~km} \mathrm{[57].}$ To implement these results in a Bayesian analysis, the function $\alpha(M, R)$ has been reconstructed with a kernel density estimation using mass-radius data from [67]. The above constraints are shown in Fig. 12.

\subsection{Fictitious radius measurements}

Simultaneous measurements of masses and radii like the ones provided recently by NICER are very important for deriving constraints on the EoS. However, due to limited observation time, the difficult decision has to be made for which object with a known mass should a precise radius measurement (and thus a long-term observation campaign) bear the largest discovery potential. A BA can support such decisions by using fictitious results of a radius measurement on the object under scrutiny. An essential input for the BA would be precise radius measurements for pulsars with known masses. One example is the high-mass millisecond pulsar PSR J0740+6620, for which the mass is known from Ref. [54] with the recent update by [55], but for which by the time this paper was written the results of the NICER radius measurement were not yet available. Now, after their publication in Refs. [56,57], we can compare the these real results with the predictions of the fictitious radii and suggest the method of fictitious radii for supporting decisions on the selection of targets for future measurements. We consider here three different values for the radius of PSR J0740+6620,

\footnotetext{
${ }^{3}$ Z. Arzoumanian \& K. C. Gendreau. Focus on NICER Constraints on the Dense Matter Equation of State, ApJ 887, 2019.
}

namely $R=11,12$ and $13 \mathrm{~km}$ with the same designed uncertainty $\sigma=0.5 \mathrm{~km}$ of the NICER experiment. The likelihood for fictitious measurements is the same as introduced above (23).

\subsection{Sets of constraints}

We suggest three sets of constraints:

1: the mass measurement for PSR J0740+6620 [55] as the lower limit for the maximum mass, the tidal deformability from GW170817 [4] and the mass-radius constraint from PSR J0030+0451 [58] (set 1);

2: in addition to the constraints of set 1 , the constraint on the upper limit of the maximum mass from Ref. [60] is included;

$\underline{3}$ : as for set 1 , but assuming that the lower mass companion of the black hole in the asymmetric binary merger GW190814 [3] was a neutron star, the lower limit for the maximum mass is replaced by the lower limit on its mass $M_{190814}=2.59_{-0.09}^{+0.08} M_{\odot}$.

Besides these "pure" sets of constraints, we investigate for each of them the possibility of an additional mass-radius constraint for the pulsar PSR J0740+6620, for which the mass $2.08 \pm 0.07 M_{\odot}$ is rather precisely measured and a result for the radius measurement by the NICER experiment is anticipated to yield the values of 11,12 and $13 \mathrm{~km}$. We denote these subsets with the numbers 1, 2 and 3, respectively. The entirety of Bayesian constraint sets in this work is synoptically summarized in Table 1. A separate BA is performed using the recent measurement of the combined mass-radius constraint for PSR J0740+6620 on the basis of NICER and XMM Newton data, and compared to the predictions using fictitious radii.

\section{Results of the Bayesian analysis}

In this section we show and discuss our results for the BA of the hybrid EoS for compact stars under the constraints defined in the previous section and summarized in Table 1. The details of the considered families of hybrid EoS, the interpolation method, the constant speed of sound representation, the BA and astrophysical constraints were already presented in previous sections.

Figure 15 shows the results of our BA for the $\eta_{V}$ and $\eta_{D}$ values of our EoS models under consideration. The difference between the three LEGO plots is that for their derivation the values of the maximum mass constraint have been changed. These three different cases comprise (1) the mass measurement of the object PSR J0740+6620, [55], (2) the previous measurement plus the upper limit of the maximum 
Table 1 Overview on Bayesian analysis constraints employed in the present work. The rightmost column refers to the set associated figure number in the text and posterior probability LEGO plot position in it according to the following convention: columns are indicated by left $(1)$, center (c), and right ( $\mathrm{r})$, while rows are labelled upper (u), middle (m), and bottom (b)

\begin{tabular}{|c|c|c|c|c|c|c|c|c|c|}
\hline \multirow[t]{4}{*}{ Set } & \multicolumn{3}{|c|}{ Maximum mass $\left[\mathrm{M}_{\odot}\right]$} & \multirow{4}{*}{$\begin{array}{l}M-R \\
\text { J0030+0451 [58] }\end{array}$} & \multirow{4}{*}{$\frac{\Lambda_{1}-\Lambda_{2}}{\text { GW170817 [4] }}$} & \multicolumn{3}{|c|}{$\begin{array}{l}\text { Fictitious radius measurement on PSR } \\
\mathrm{J} 0740+6620[\mathrm{~km}]\end{array}$} & \multirow[t]{4}{*}{ Figure } \\
\hline & \multicolumn{2}{|c|}{ Lower limit } & \multirow{3}{*}{$\begin{array}{l}\text { Upper limit } \\
\text { GW170817 } \\
2.16 \pm 0.17[60]\end{array}$} & & & & & & \\
\hline & PSR J0740+6620 & GW190814 & & & & & & & \\
\hline & $2.08 \pm 0.07[55]$ & $2.59 \pm 0.09[3]$ & & & & $11 \pm 0.5$ & $12 \pm 0.5$ & $13 \pm 0.5$ & \\
\hline 1.0 & $\checkmark$ & & & $\checkmark$ & $\checkmark$ & & & & $15(1)$ \\
\hline 1.1 & & & & $\checkmark$ & $\checkmark$ & $\checkmark$ & & & $16(1, \mathrm{u})$ \\
\hline 1.2 & & & & $\checkmark$ & $\checkmark$ & & $\checkmark$ & & $16(\mathrm{c}, \mathrm{u})$ \\
\hline 1.3 & & & & $\checkmark$ & $\checkmark$ & & & $\checkmark$ & $16(\mathrm{r}, \mathrm{u})$ \\
\hline 2.0 & $\checkmark$ & & $\checkmark$ & $\checkmark$ & $\checkmark$ & & & & $15(\mathrm{c})$ \\
\hline 2.1 & & & $\checkmark$ & $\checkmark$ & $\checkmark$ & $\checkmark$ & & & $16(1, \mathrm{~m})$ \\
\hline 2.2 & & & $\checkmark$ & $\checkmark$ & $\checkmark$ & & $\checkmark$ & & $16(\mathrm{c}, \mathrm{m})$ \\
\hline 2.3 & & & $\checkmark$ & $\checkmark$ & $\checkmark$ & & & $\checkmark$ & $16(\mathrm{r}, \mathrm{m})$ \\
\hline 3.0 & $\checkmark$ & $\checkmark$ & & $\checkmark$ & $\checkmark$ & & & & $15(\mathrm{r})$ \\
\hline 3.1 & & $\checkmark$ & & $\checkmark$ & $\checkmark$ & $\checkmark$ & & & $16(1, b)$ \\
\hline 3.2 & & $\checkmark$ & & $\checkmark$ & $\checkmark$ & & $\checkmark$ & & $16(c, b)$ \\
\hline 3.3 & & $\checkmark$ & & $\checkmark$ & $\checkmark$ & & & $\checkmark$ & $16(r, b)$ \\
\hline
\end{tabular}
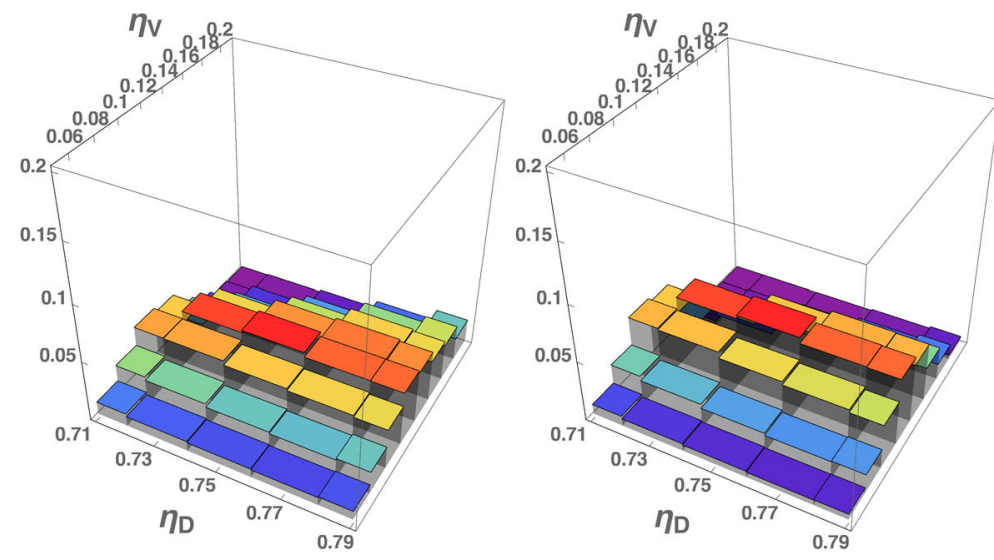

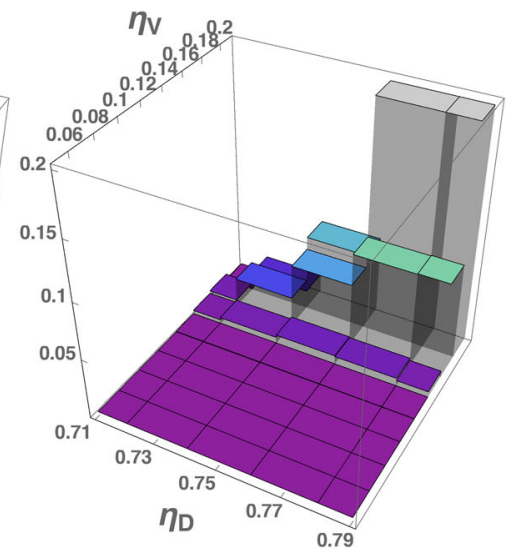

Fig. 15 Bayesian analysis using the mass measurement for PSR J0740+6620 [55] as the lower limit for the maximum mass, the tidal deformability from GW170817 [4] and the mass-radius constraint from PSR J0030+0451 for the class of hybrid EoS obtained with a twozone interpolation between APR and nINJL in the two-dimensional EoS parameter plane spanned by $\eta_{V}$ and $\eta_{D}$ (left panel; set 1 ). In the middle panel the constraint on the upper limit of the maximum mass from Ref. [60] has been added (set 2) and in the right panel this limit has been lifted again in favor of the new lower limit on the maximum mass from lower-mass companion of the black hole in the asymmetric binary merger GW190814 [3] that replaces the one from [55] (set 3), if the former object would not be a black hole mass from Ref. [60], (3) the first measurement plus the mass for the lighter object in the binary merger GW190814 [3] assuming that it was a neutron star. We can see that whereas for the first to cases the posterior probability distributions peak at intermediate values of the $\eta_{V}$ parameter of quark matter, the third case favours its highest values. The probability distribution in $\eta_{D}$ direction remains flat for the three cases.

The situation becomes more interesting when we consider a new, fictitious mass and radius measurement which antic- ipates the outcome of the NICER observation of the heavy pulsar PSR J0740+6620. In Fig. 16 each column corresponds to the results for such a radius measurement with a value of $R=11 \mathrm{~km}, R=12 \mathrm{~km}$ or $R=13 \mathrm{~km}$, whereas the rows represent the same three aforementioned cases for the constraints. Just like before, (case 3) displays a more selective effect on the $\eta_{V}$ parameter, however the inclusion of this new radius observation favours the highest $\eta_{D}$ values for most of the plots. 


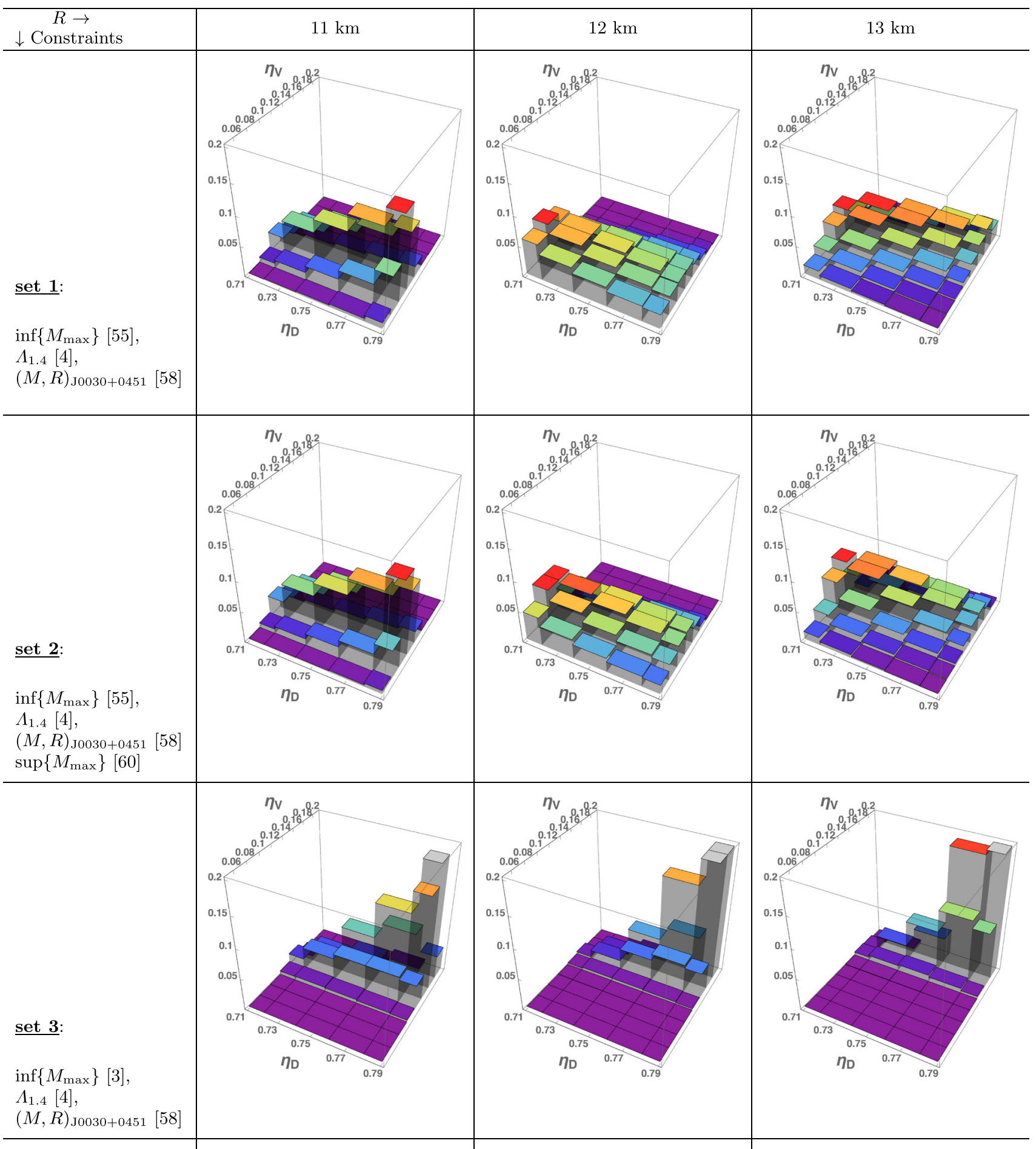

Fig. 16 Probabilities when an additional measurement of the radius $R$ of PSR J0740+6220 (as provided recently by the NICER experiment [56]) is taken into account with anticipated values of $R=11 \mathrm{~km}$ (second column), $12 \mathrm{~km}$ (third column) or $13 \mathrm{~km}$ (fourth column) and with a standard deviation of $\sigma_{R}=0.5 \mathrm{~km}$. The mass is taken from the measurement by Fonseca et al. [55]. The three rows correspond to set 1, set 2 and set 3 , respectively 


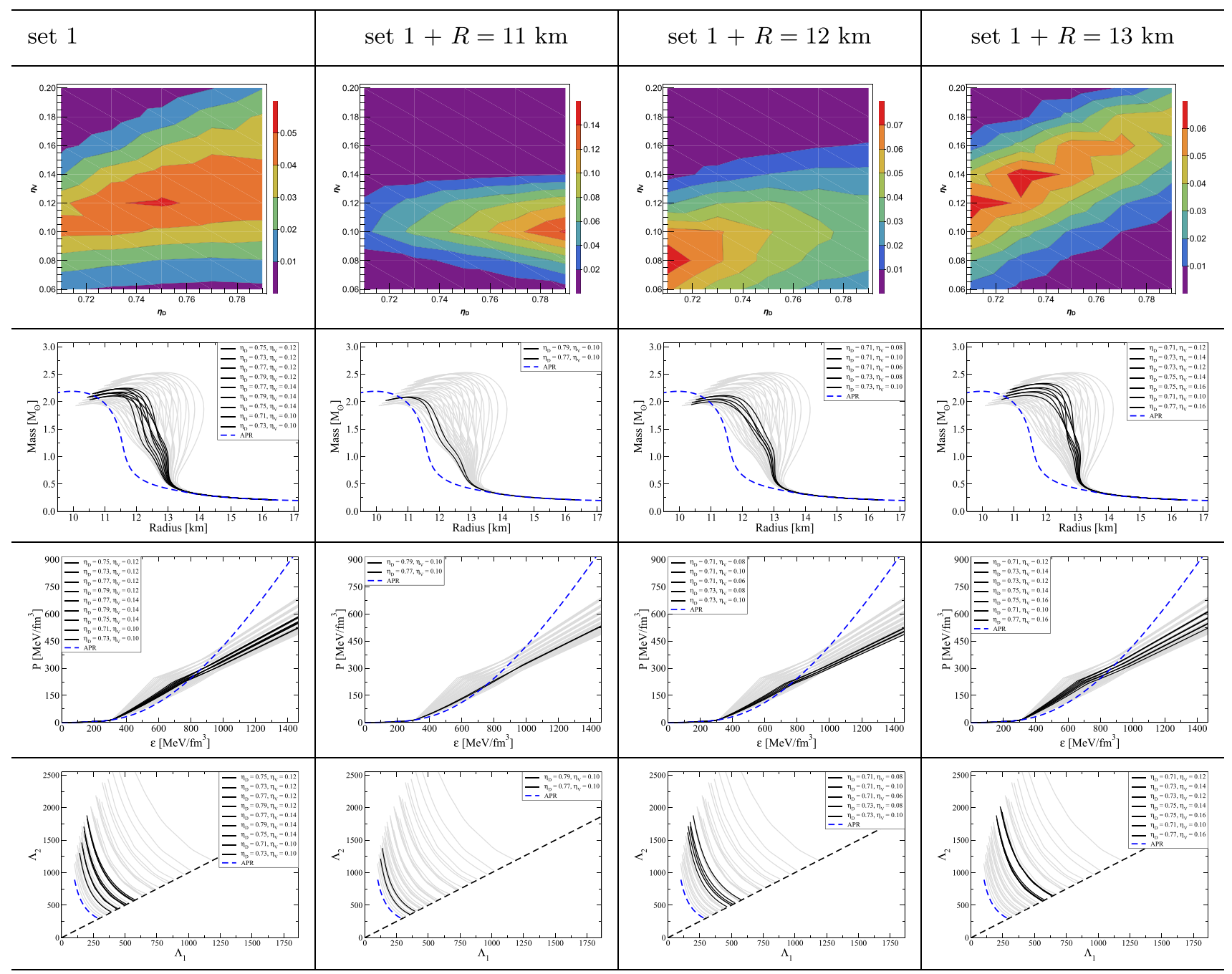

Fig. 17 Results of the BA for set 1 which includes the constraints $\left(\inf \left\{M_{\max }\right\}\right.$ [55], $\Lambda_{1.4}$ [4], $(M, R)_{\mathrm{J} 0030+0451}$ and [58]) in the leftmost column and with an additional (yet fictitious) NICER radius measurement for PSR J0740+6620 of $R=11,12$ or $13 \mathrm{~km}$ with an estimated standard deviation of $\sigma_{R}=0.5 \mathrm{~km}$ in the other three columns. The highlighted most probable M-R sequences (2nd row), EoS (3rd row) and $\Lambda_{1}-\Lambda_{2}$ (4th row) relationships correspond to the parameter sets with at least $75 \%$ of the maximum probability as shown in the first row

tious radii anticipation can be considered a reliable tool in predicting the implications of future $M-R$ measurements, e.g., by the NICER collaboration. Moreover, for the most probable parameter set, the corresponding EoS lead to the $M-R$ sequences highlighted in Fig. 20 which fulfill the $2 M_{\odot}$ mass constraint but do not reach masses above $2.5 M_{\odot}$ which would be required if the lighter object in GW190814 was a (hybrid) neutron star.

\section{Conclusions}

In the present work we have applied Bayesian analysis methods to investigate the most likely quark-hadron hybrid EoS 


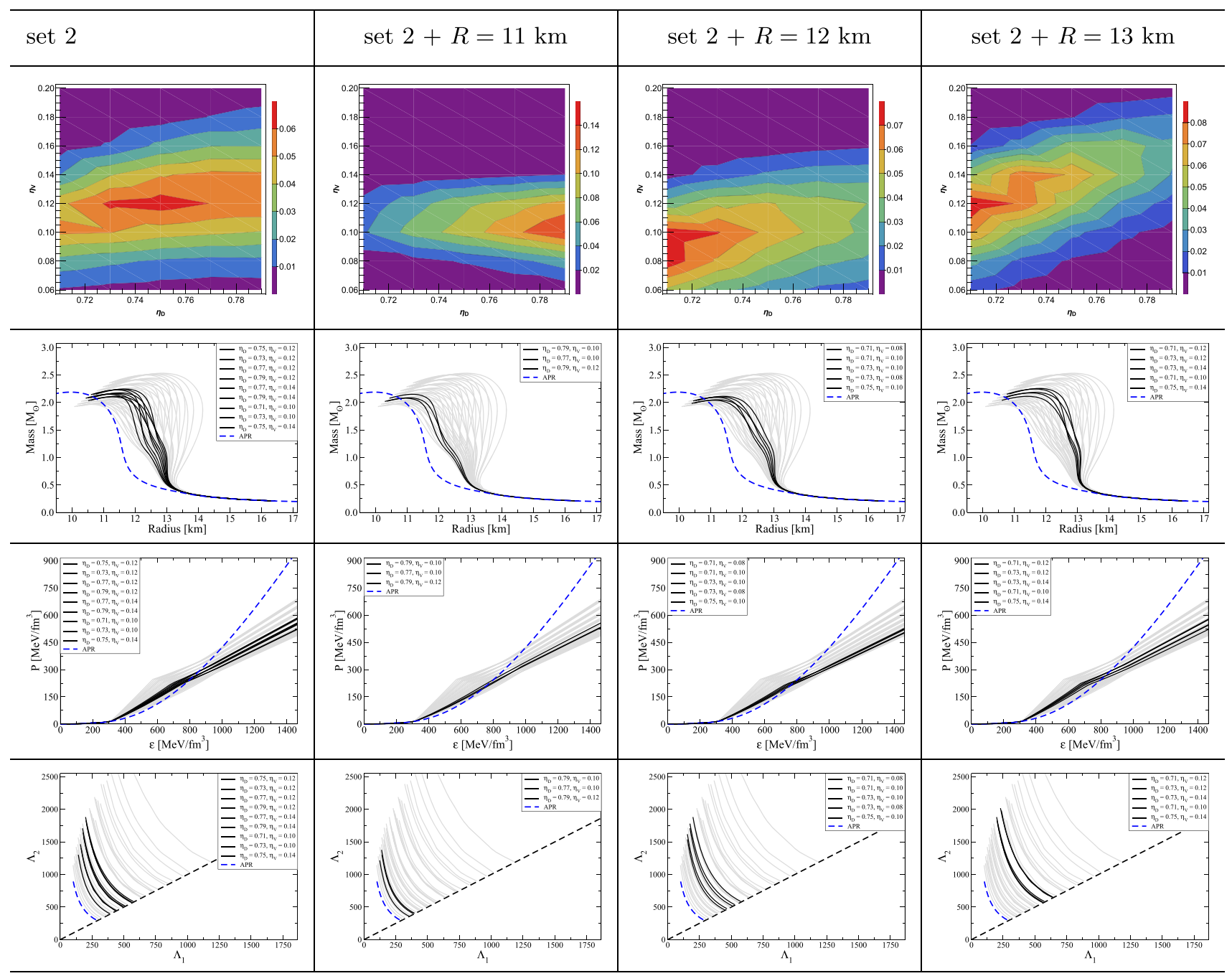

Fig. 18 Results of the BA for set 2 which includes the constraints (inf $\left\{M_{\max }\right\}$ [55], $\Lambda_{1.4}$ [4], $(M, R)_{\mathrm{J} 0030+0451}$ [58], $\sup \left\{M_{\max }\right\}$ [60]) in the leftmost column and with an additional (yet fictitious) NICER radius measurement for PSR J0740+6620 of $R=11,12$ or $13 \mathrm{~km}$ with an estimated standard deviation of $\sigma_{R}=0.5 \mathrm{~km}$ in the other three columns.
The highlighted most probable M-R sequences (2nd row), EoS (3rd row) and $\Lambda_{1}-\Lambda_{2}$ (4th row) relationships correspond to the parameter sets with at least $75 \%$ of the maximum probability as shown in the first row

sity, setting the corresponding parameter $\Delta n=0$. In future work, we plan to systematically investigate also the extension of the new two-zone interpolation construction to the cases with $\Delta n \neq 0$. We note, however, that the interpolation construction may be viewed as a shortcut that replaces three microphysical effects: (1) stiffening of the nuclear EoS due to quark substructure effects (quark Pauli blocking modeled, e.g., by a baryon excluded volume), (2) softening of the quark matter EoS at low densities due to confining effects (modeled, e.g., by a medium-dependent bag pressure) and (3) mixedphase effects due to the occurrence of finite-size structures (pasta phases). This possible microphysical underpinning of the interpolation construction, in particular within the twozone version suggested in this paper, has been discussed and 


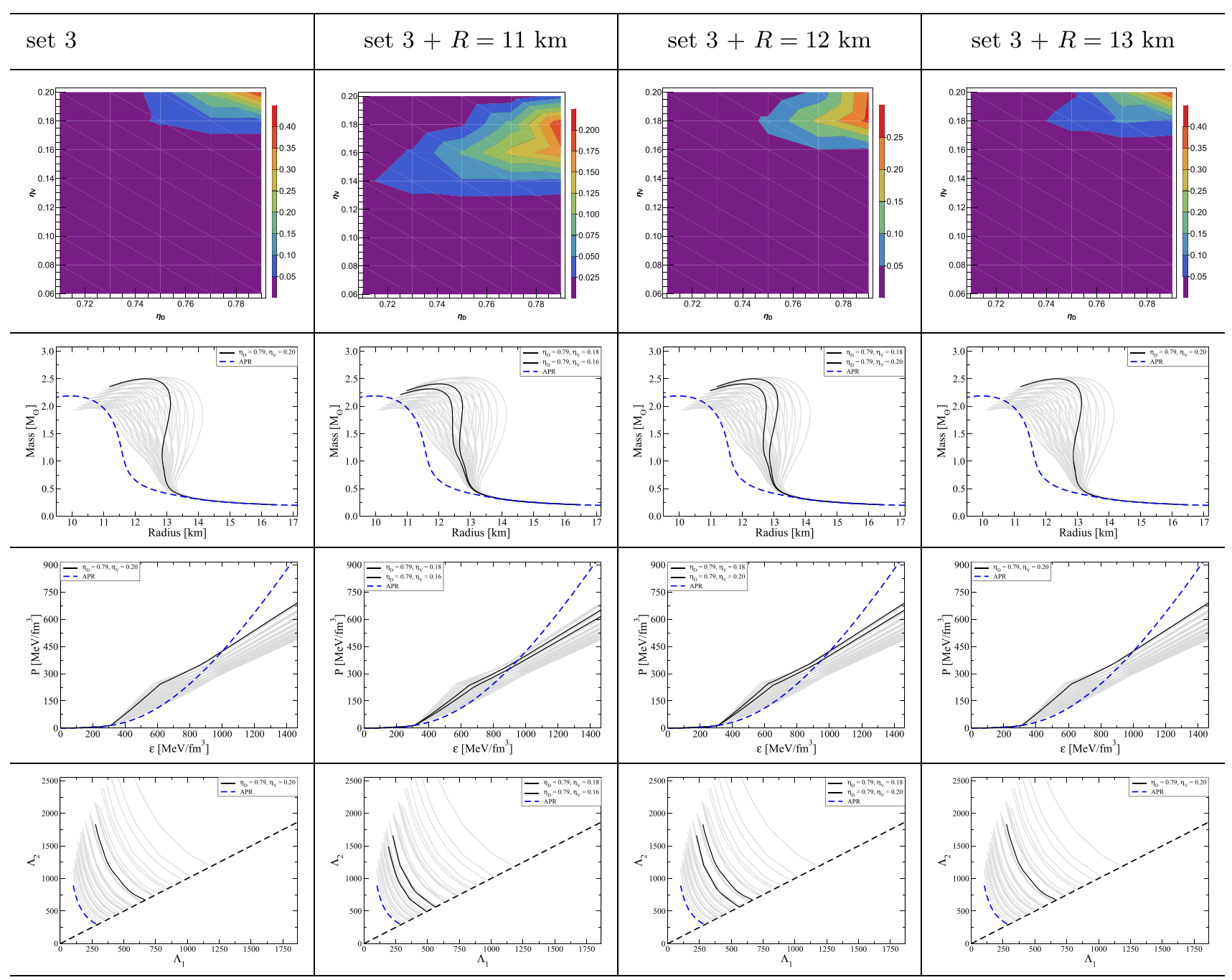

Fig. 19 Results of the BA for set 3 of constraints $\left(\inf \left\{M_{\max }\right\}\right.$ [3], $\Lambda_{1.4}$ [4], $\left.(M, R)_{\mathrm{J} 0030+0451}[58]\right)$ in the leftmost column and with an additional (yet fictitious) NICER radius measurement for PSR J0740+6620 of $R=11,12$ or $13 \mathrm{~km}$ with an estimated standard deviation of
$\sigma_{R}=0.5 \mathrm{~km}$ in the other three columns. The highlighted most probable M-R sequences (2nd row), EoS (3rd row) and $\Lambda_{1}-\Lambda_{2}$ (4th row) relationships correspond to the parameter sets with at least $75 \%$ of the maximum probability as shown in the first row
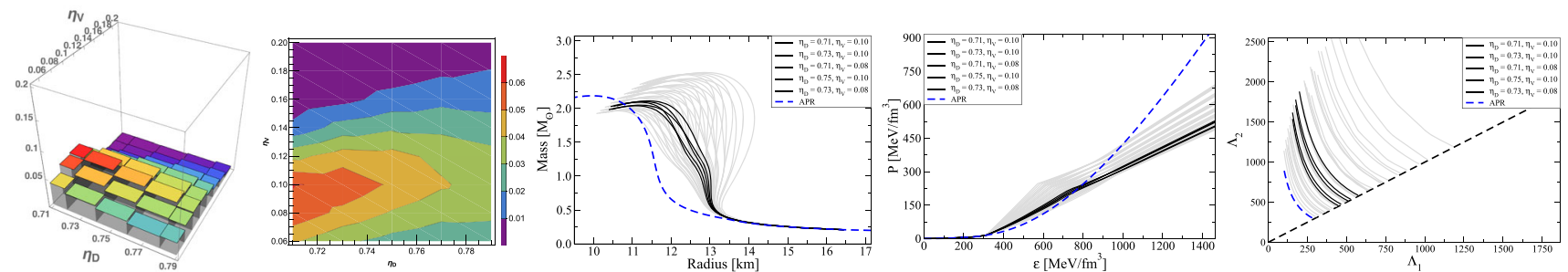

Fig. 20 Bayesian analysis considering $\Lambda_{1}-\Lambda_{2}$ from GW170817, M-R from J0030+0451 and M-R from J0740+6620 measurements 
illustrated in the Introduction, but a detailed exploration is deferred to future work.

In our Bayesian study we have applied standard constraints for mass and radius measurements in set 1 and demonstrated their effect of narrowing the viable range of parameter values. We obtained the result that the most probable EoS lie along a line of proportionality between $\eta_{V}$ and $\eta_{D}$, whereby the higher values of $\eta_{V}$ are favorable for obtaining larger maximum masses of hybrid neutron stars. This finding confirms similar results of earlier studies in $[40,68]$ and the recent work [25] which do not employ Bayesian methods.

In the sets 2 and 3 we explored the nonstandard constraints of an upper limit on the maximum mass and the high mass of the lighter companion object of GW190814 as a lower limit on the maximum mass, respectively. While for the set 2 the narrowing of the parameter range due to the upper limit on the maximum mass leads to an exclusion of the higher values of the vector meson coupling, the high value of the lower limit on the maximum mass for set 3 allows only the highest possible vector couplings resulting in stiffer EoS and thus larger maximum masses and radii. It is a remarkable fact that within the present interpolation approach the $2.6 M_{\odot}$ companion object in GW190814 could be a hybrid star with quark matter core. This possibility has been pointed out before in several works, among them $[45,69,70]$.

Finally, we have used the Bayesian approach to explore the consequences that the radius measurements on the $2 M_{\odot}$ pulsar PSR J0740+6620 by the NICER experiment may have for neutron star phenomenology.

Before the NICER radius measurements on J0740+6620 $[56,57]$ were released, we have performed Bayesian analyses with fictitious results for that radius measurement, anticipating $11 \mathrm{~km}, 12 \mathrm{~km}$ and $13 \mathrm{~km}$ as a possible outcome. For a radius value as small as $11 \mathrm{~km}$ or less, the hybrid star scenario for the $2.6 M_{\odot}$ object in GW190814 could be excluded and this event was a binary black hole merger. In that case the present two-zone interpolation approach with continuous crossover is not suitable since it does not produce stable hybrid stars with small enough radii above $2 M_{\odot}$.

For a radius of $\mathrm{J} 0740+6620$ as large as $13 \mathrm{~km}$ or more, the likely interpretation of GW190814 is that of a neutron star - black hole merger where the neutron star possibly had a color superconducting quark matter core.

We have performed a Bayesian analysis with the actual results for the NICER radius measurement on PSR J0740+ 6620 as reported by the Maryland-Illinois team [56] and compared the outcome for the probability contours in the model parameter space with those for the fictitious radius measurements. A striking similarity was obtained for $R=12 \mathrm{~km}$, which proves the usefulness of the fictitious radius method for estimating the outcome of future NICER radius measurement campaigns and its impact for the dense matter EoS and neutron star phenomenology.
The outcome of the Bayesian analysis in our setting favors the scenario that GW190814 was a binary black hole merger and not a neutron star - black hole merger. The favored EoS which are highlighted in Fig. 20 favor $M-R$ sequences with a maximum mass that lies well below $2.5 M_{\odot}$, as shown also in that figure.

In future extensions of the Bayesian approach to neutron star phenomenology it is desirable to widen the class of EoS by extending the present direct smooth interpolation approach to a first-order phase transition scenario [11] where in the present two-zone interpolation method a nonzero density jump parameter at the matching point would be chosen, and to augment this with the possibility of a subsequent smoothing of the transition by a pasta phase construction or its emulation.

In order to compare the results of two Bayesian analyzes obtained on the same astrophysical data, it is necessary to combine the factors of the normalization of the Bayesian formula (18). This allows to introduce the relative posterior probability of each analysis. The set of EoS models, whose analysis gives a greater value of the relative posterior probability can be considered to be more successful in describing the observational data. A comparison of the result presented in this paper with the previous result from Ref. [11], where constraints corresponding to set 1 have been employed, shows that the likelihood for models considered in [11] exceeds that of the present work by more than 5 times. Such a direct comparison is possible only for the case of set 1 , because the other two sets have not been considered in [11].

The question has been raised whether one should generalize the Bayesian analysis by sytematically varying also the hadronic EoS at supersaturation densities like, e.g., within an excluded volume approach (see Ref. [71]). In the present setting of the two-zone interpolation model, with a choice of the onset of the interpolated part of the EoS at the saturation density $\left(n_{H}=n_{0}\right)$, there is no room for such a variation. Uncertainties in the knowledge of the nuclear EoS at supersaturation densities are covered by the variation of the parameters in the hadron-like zone of the interpolation.

Acknowledgements We acknowledge the partial support by the COST Action CA16214 "PHAROS" for our international networking activities in preparing this article. This work received support from the Russian Foundation for Basic Research under grant no. 18-02-40137. The work by D.B. on the new class of quark-hadron hybrid EoS in Section 4 was supported by the Polish National Science Centre under grant number UMO 2019/33/B/ST9/03059. This work is part of a project that has received funding from the European Union's Horizon 2020 research and innovation program under grant agreement STRONG - 2020 - No 824093. D.E.A-C. and H.G. are grateful for support from the programme for exchange between JINR Dubna and Polish Institutes (BogoliubovInfeld programme). A. G. G. would like to acknowledge to CONICET for financial support under Grant No. PIP17-700. 
Data Availability Statement This manuscript has no associated data or the data will not be deposited. [Authors' comment: Data is available upon request from the authors.]

Open Access This article is licensed under a Creative Commons Attribution 4.0 International License, which permits use, sharing, adaptation, distribution and reproduction in any medium or format, as long as you give appropriate credit to the original author(s) and the source, provide a link to the Creative Commons licence, and indicate if changes were made. The images or other third party material in this article are included in the article's Creative Commons licence, unless indicated otherwise in a credit line to the material. If material is not included in the article's Creative Commons licence and your intended use is not permitted by statutory regulation or exceeds the permitted use, you will need to obtain permission directly from the copyright holder. To view a copy of this licence, visit http://creativecomm ons.org/licenses/by/4.0/.

\section{References}

1. B. Abbott et al. [LIGO Scientific and Virgo], GW170817: observation of gravitational waves from a binary neutron star inspiral. Phys. Rev. Lett. 119(16), 161101 (2017)

2. B. P. Abbott et al. [LIGO Scientific, Virgo, Fermi GBM, INTEGRAL, IceCube, AstroSat Cadmium Zinc Telluride Imager Team, IPN, Insight-Hxmt, ANTARES, Swift, AGILE Team, 1M2H Team, Dark Energy Camera GW-EM, DES, DLT40, GRAWITA, FermiLAT, ATCA, ASKAP, Las Cumbres Observatory Group, OzGrav, DWF (Deeper Wider Faster Program), AST3, CAASTRO, VINROUGE, MASTER, J-GEM, GROWTH, JAGWAR, CaltechNRAO, TTU-NRAO, NuSTAR, Pan-STARRS, MAXI Team, TZAC Consortium, KU, Nordic Optical Telescope, ePESSTO, GROND, Texas Tech University, SALT Group, TOROS, BOOTES, MWA, CALET, IKI-GW Follow-up, H.E.S.S., LOFAR, LWA, HAWC, Pierre Auger, ALMA, Euro VLBI Team, Pi of Sky, Chandra Team at McGill University, DFN, ATLAS Telescopes, High Time Resolution Universe Survey, RIMAS, RATIR and SKA South Africa/MeerKAT], Multi-messenger observations of a binary neutron star merger. Astrophys. J. Lett. 848(2), L12 (2017)

3. R. Abbott et al. [LIGO Scientific and Virgo], GW190814: gravitational waves from the coalescence of a 23 solar mass black hole with a 2.6 solar mass compact object. Astrophys. J. Lett. 896(2), L44 (2020)

4. B. Abbott et al. [LIGO Scientific and Virgo], GW170817: measurements of neutron star radii and equation of state. Phys. Rev. Lett. 121(16), 161101 (2018)

5. C.D. Capano, I. Tews, S.M. Brown, B. Margalit, S. De, S. Kumar, D.A. Brown, B. Krishnan, S. Reddy, Stringent constraints on neutron-star radii from multimessenger observations and nuclear theory. Nat. Astron. 4(6), 625-632 (2020)

6. D. Blaschke, H. Grigorian, G. Röpke, Chirally improved quark Pauli blocking in nuclear matter and applications to quark deconfinement in neutron stars. Particles 3(2), 477-499 (2020)

7. K. Fukushima, T. Kojo, W. Weise, Hard-core deconfinement and soft-surface delocalization from nuclear to quark matter. Phys. Rev. D 102(9), 096017 (2020)

8. G. Baym, T. Hatsuda, T. Kojo, P. D. Powell, Y. Song, T. Takatsuka, From hadrons to quarks in neutron stars: a review. Rept. Prog. Phys. 81(5), 056902 (2018)

9. N.K. Glendenning, First order phase transitions with more than one conserved charge: consequences for neutron stars. Phys. Rev. D 46, 1274-1287 (1992)

10. K. Maslov, N. Yasutake, A. Ayriyan, D. Blaschke, H. Grigorian, T. Maruyama, T. Tatsumi, N. Voskresensky, Hybrid equation of state with pasta phases and third family of compact stars. Phys. Rev. C 100(2), 025802 (2019)

11. D. Blaschke, A. Ayriyan, D. E. Alvarez-Castillo, H. Grigorian, Was GW170817 a canonical neutron star merger? Bayesian analysis with a third family of compact stars. Universe 6(6), 81 (2020)

12. U.H. Gerlach, Equation of state at supranuclear densities and the existence of a third family of superdense stars. Phys. Rev. 172, 1325-1330 (1968)

13. N.K. Glendenning, C. Kettner, Nonidentical neutron star twins. Astron. Astrophys. 353, L9 (2000)

14. A. Akmal, V. Pandharipande, D. Ravenhall, The equation of state of nucleon matter and neutron star structure. Phys. Rev. C 58, 18041828 (1998)

15. D.E. Alvarez-Castillo, D.B. Blaschke, A.G. Grunfeld, V.P. Pagura, Phys. Rev. D 99(6), 063010 (2019)

16. J.L. Zdunik, P. Haensel, Maximum mass of neutron stars and strange neutron-star cores. Astron. Astrophys. 551, A61 (2013)

17. M. Alford, M. Braby, M.W. Paris, S. Reddy, Hybrid stars that masquerade as neutron stars. Astrophys. J. 629, 969 (2005)

18. D. Blaschke, D.E. Alvarez-Castillo, High-mass twins \& resolution of the reconfinement, masquerade and hyperon puzzles of compact star interiors. AIP Conf. Proc. 1701, 020013 (2016)

19. H. Satz, The States of Strongly Interacting Matter (Springer, Heidelberg, 2010)

20. T. Schäfer, F. Wilczek, Continuity of quark and hadron matter. Phys. Rev. Lett. 82, 3956-3959 (1999)

21. T. Schäfer, F. Wilczek, Quark description of hadronic phases. Phys. Rev. D 60, 074014 (1999)

22. C. Wetterich, Gluon meson duality. Phys. Lett. B 462, 164-168 (1999)

23. T. Hatsuda, M. Tachibana, N. Yamamoto, G. Baym, New critical point induced by the axial anomaly in dense QCD. Phys. Rev. Lett. 97, 122001 (2006)

24. N. Yamamoto, M. Tachibana, T. Hatsuda, G. Baym, Phase structure, collective modes, and the axial anomaly in dense QCD. Phys. Rev. D 76, 074001 (2007)

25. G. Baym, S. Furusawa, T. Hatsuda, T. Kojo, H. Togashi, New neutron star equation of state with Quark-Hadron crossover. Astrophys. J. 885, 42 (2019)

26. K. Masuda, T. Hatsuda, T. Takatsuka, Hadron-Quark crossover and massive hybrid stars with strangeness. Astrophys. J. 764, 12 (2013)

27. K. Masuda, T. Hatsuda, T. Takatsuka, Hadron-Quark crossover and massive hybrid stars. PTEP 2013(7), 073D01 (2013)

28. K. Masuda, T. Hatsuda, Hyperon puzzle, Hadron-Quark crossover and massive neutron stars. Eur. Phys. J. A 52(3), 65 (2016)

29. D. L. Whittenbury, H. H. Matevosyan, A. W. Thomas, Hybrid stars using the quark-meson coupling and proper-time Nambu-JonaLasinio models. Phys. Rev. C 93(3), 035807 (2016)

30. K. Hebeler, J.M. Lattimer, C.J. Pethick, A. Schwenk, Equation of state and neutron star properties constrained by nuclear physics and observation. Astrophys. J. 773, 11 (2013)

31. A. Kurkela, E.S. Fraga, J. Schaffner-Bielich, A. Vuorinen, Constraining neutron star matter with quantum chromodynamics. Astrophys. J. 789, 127 (2014)

32. E. S. Fraga, A. Kurkela, A. Vuorinen, Neutron star structure from QCD. Eur. Phys. J. A 52(3), 49 (2016)

33. E. Annala, T. Gorda, A. Kurkela, A. Vuorinen, Gravitational-wave constraints on the neutron-star-matter Equation of State. Phys. Rev. Lett. 120(17), 172703 (2018)

34. V. Paschalidis, K. Yagi, D. Alvarez-Castillo, D. B. Blaschke, A. Sedrakian, Implications from GW170817 and I-Love-Q relations for relativistic hybrid stars. Phys. Rev. D 97(8), 084038 (2018)

35. S. K. Greif, K. Hebeler, J. M. Lattimer, C. J. Pethick, A. Schwenk, Equation of state constraints from nuclear physics, neutron star masses, and future moment of inertia measurements. Astrophys. J. 901(2), 155 (2020) 
36. M. C. Miller, C. Chirenti, F. K. Lamb, Constraining the equation of state of high-density cold matter using nuclear and astronomical measurements. Astrophys. J. 888(1), 12 (2020)

37. F. Douchin, P. Haensel, J. Meyer, Nuclear surface and curvature properties for SLy Skyrme forces and nuclei in the inner neutronstar crust. Nucl. Phys. A 665, 419 (2000)

38. D.B. Blaschke, D. Gomez Dumm, A.G. Grunfeld, T. Klähn, N.N. Scoccola, Hybrid stars within a covariant, nonlocal chiral quark model. Phys. Rev. C 75, 065804 (2007)

39. D.S. Zablocki, D.B. Blaschke, R. Anglani, Y.L. Kalinovsky, Diquark Bose-Einstein condensation at strong coupling. Acta Phys. Polon. Supp. 3, 771-776 (2010)

40. T. Klähn, R. Łastowiecki, D. B. Blaschke, Implications of the measurement of pulsars with two solar masses for quark matter in compact stars and heavy-ion collisions: a Nambu-Jona-Lasinio model case study. Phys. Rev. D 88(8), 085001 (2013)

41. A. Ayriyan, N. U. Bastian, D. Blaschke, H. Grigorian, K. Maslov, D. N. Voskresensky, Robustness of third family solutions for hybrid stars against mixed phase effects. Phys. Rev. C 97(4), 045802 (2018)

42. V. Abgaryan, D. Alvarez-Castillo, A. Ayriyan, D. Blaschke, H. Grigorian, Two novel approaches to the Hadron-Quark mixed phase in compact stars. Universe 4(9), 94 (2018)

43. S. Antić, M. Shahrbaf, D. Blaschke and A. G. Grunfeld, Parameter mapping between the microscopic nonlocal Nambu-Jona-Lasinio and constant-sound-speed models, arXiv:2105.00029 [nucl-th]

44. M. G. Alford, S. Han, M. Prakash, Generic conditions for stable hybrid stars. Phys. Rev. D 88(8), 083013 (2013)

45. D. Blaschke, M. Cierniak, Studying the onset of deconfinement with multi-messenger astronomy of neutron stars. Astron. Nachr. 342(1-2), 227-233 (2021)

46. L. McLerran, S. Reddy, Quarkyonic matter and neutron stars. Phys. Rev. Lett 122(12), 122701 (2019)

47. R.C. Tolman, Static solutions of Einstein's field equations for spheres of fluid. Phys. Rev. 55, 364 (1939)

48. J.R. Oppenheimer, G.M. Volkoff, On massive neutron cores. Phys. Rev. 55, 374 (1939)

49. T. Hinderer, Tidal Love numbers of neutron stars. Astrophys. J. 677, $1216(2008)$

50. T. Damour, A. Nagar, Relativistic tidal properties of neutron stars. Phys. Rev. D 80, 084035 (2009)

51. T. Binnington, E. Poisson, Relativistic theory of tidal Love numbers. Phys. Rev. D 80, 084018 (2009)

52. K. Yagi, N. Yunes, I-Love-Q relations in neutron stars and their applications to astrophysics, gravitational waves and fundamental physics. Phys. Rev. D 88(2), 023009 (2013)

53. T. Hinderer, B.D. Lackey, R.N. Lang, J.S. Read, Tidal deformability of neutron stars with realistic equations of state and their gravitational wave signatures in binary inspiral. Phys. Rev. D 81, $123016(2010)$

54. H.T. Cromartie, E. Fonseca, S.M. Ransom, P.B. Demorest, Z. Arzoumanian, H. Blumer, P.R. Brook, M.E. DeCesar, T. Dolch, J.A. Ellis, R.D. Ferdman, E.C. Ferrara, N. Garver-Daniels, P.A. Gentile, M.L. Jones, M.T. Lam, D.R. Lorimer, R.S. Lynch, M.A. McLaughlin, C. Ng, D.J. Nice, T.T. Pennucci, R. Spiewak, I.H. Stairs, K. Stovall, J.K. Swiggum, W. Zhu, Relativistic Shapiro delay measurements of an extremely massive millisecond pulsar. Nat. Astron. 4(1), 72-76 (2019)

55. E. Fonseca, H.T. Cromartie, T.T. Pennucci, P.S. Ray, A.Y. Kirichenko, S.M. Ransom, P.B. Demorest, I.H. Stairs, Z. Arzoumanian, L. Guillemot et al., Refined Mass and Geometric Measurements of the High-mass PSR J0740+6620. Astrophys. J. Lett. 915(1), L12 (2021)

56. M. C. Miller, F. K. Lamb, A. J. Dittmann, S. Bogdanov, Z. Arzoumanian, K. C. Gendreau, S. Guillot, W. C. G. Ho, J. M. Lattimer and M. Loewenstein, et al. The Radius of PSR J0740+6620 from
NICER and XMM-Newton Data. Astrophys. J. Lett. 918(2), L28 (2021)

57. T. E. Riley, A. L. Watts, P. S. Ray, S. Bogdanov, S. Guillot, S. M. Morsink, A. V. Bilous, Z. Arzoumanian, D. Choudhury and J. S. Deneva, et al. A NICER View of the Massive Pulsar PSR J0740+6620 Informed by Radio Timing and XMM-Newton Spectroscopy. Astrophys. J. Lett. 918(2), L27 (2021)

58. M. Miller, F. Lamb, A. Dittmann, S. Bogdanov, Z. Arzoumanian, K. Gendreau, S. Guillot, A. Harding, W. Ho, J. Lattimer, R. Ludlam, S. Mahmoodifar, S. Morsink, P. Ray, T. Strohmayer, K. Wood, T. Enoto, R. Foster, T. Okajima, G. Prigozhin, Y. Soong, PSR J0030+0451 mass and radius from NICER data and implications for the properties of neutron star matter. Astrophys. J. Lett. 887(1), L24 (2019)

59. A. Bauswein, O. Just, H.T. Janka, N. Stergioulas, Neutron-star radius constraints from GW170817 and future detections. Astrophys. J. Lett. 850(2), L34 (2017)

60. L. Rezzolla, E.R. Most, L.R. Weih, Using gravitational-wave observations and quasi-universal relations to constrain the maximum mass of neutron stars. Astrophys. J. Lett. 852(2), L25 (2018)

61. A. Bauswein, S. Blacker, V. Vijayan, N. Stergioulas, K. Chatziioannou, J. A. Clark, N. U. F. Bastian, D. B. Blaschke, M. Cierniak, T. Fischer, Equation of state constraints from the threshold binary mass for prompt collapse of neutron star mergers. Phys. Rev. Lett. 125(14), 141103 (2020)

62. C. Breu, L. Rezzolla, Maximum mass, moment of inertia and compactness of relativistic stars. Mon. Not. R. Astron. Soc. 459(1), 646-656 (2016)

63. D. Alvarez-Castillo, A. Ayriyan, G.G. Barnaföldi, H. Grigorian, P. Pósfay, Studying the parameters of the extended $\sigma-\omega$ model for neutron star matter. Eur. Phys. J. ST 229(22-23), 3615-3628 (2020)

64. A. Ayriyan, D. Alvarez-Castillo, D. Blaschke, H. Grigorian, Bayesian analysis for extracting properties of the nuclear equation of state from observational data including tidal deformability from GW170817. Universe 5(2), 61 (2019)

65. T.E. Riley, A.L. Watts, S. Bogdanov, P.S. Ray, R.M. Ludlam, S. Guillot, Z. Arzoumanian, C.L. Baker, A.V. Bilous, D. Chakrabarty, K.C. Gendreau, A.K. Harding, W.C. Ho, J.M. Lattimer, S.M. Morsink, T.E. Strohmayer, A N ICER view of PSR J0030+0451: millisecond pulsar parameter estimation. Astrophys. J. Lett. 887(1), L21 (2019)

66. M. C. Miller et al., NICER PSR J0030+0451 Illinois-Maryland MCMC Samples (Version 1.0.0) [Data set]. Astrophys. J. Lett. Zenodo. https://doi.org/10.5281/zenodo.3473466 (2019)

67. M. C. Miller et al., NICER PSR J0740+6620 Illinois-Maryland MCMC Samples [Data set]. Zenodo. https://doi.org/10.5281/ zenodo.4670689 (2021)

68. T. Klähn, D. Blaschke, F. Sandin, C. Fuchs, A. Faessler, H. Grigorian, G. Röpke, J. Trümper, Modern compact star observations and the quark matter equation of state. Phys. Lett. B 654, 170-176 (2007)

69. H. Tan, J. Noronha-Hostler, N. Yunes, Neutron star equation of state in light of GW190814. Phys. Rev. Lett. 125(26), 261104 (2020)

70. V. Dexheimer, R. O. Gomes, T. Klähn, S. Han, M. Salinas, GW190814 as a massive rapidly-rotating neutron star with exotic degrees of freedom. Phys. Rev. C 103(2), 025808 (2021)

71. D. Alvarez-Castillo, A. Ayriyan, S. Benic, D. Blaschke, H. Grigorian, S. Typel, New class of hybrid EoS and Bayesian M-R data analysis. Eur. Phys. J. A 52(3), 69 (2016) 